\title{
The Euclidean Gravitational Action as Black Hole Entropy, Singularities and Spacetime Voids
}

\author{
Carlos Castro
}

May 2007

Center for Theoretical Studies of Physical Systems, Clark Atlanta University, Atlanta; castro@ctsps.cau.edu

\begin{abstract}
We argue why the static spherically symmetric (SSS) vacuum solutions of Einstein's equations described by the textbook Hilbert metric $g_{\mu \nu}(r)$ is not diffeomorphic to the metric $g_{\mu \nu}(|r|)$ corresponding to the gravitational field of a point mass delta function source at $r=0$. By choosing a judicious radial function $R(r)=r+2 G|M| \Theta(r)$ involving the Heaviside step function, one has the correct boundary condition $R(r=0)=0$, while displacing the horizon from $r=2 G|M|$ to a location arbitrarily close to $r=0$ as one desires, $r_{h} \rightarrow 0$, where stringy geometry and quantum gravitational effects begin to take place. We solve the field equations due to a delta function point mass source at $r=0$, and show that the Euclidean gravitational action (in $\hbar$ units) is precisely equal to the black hole entropy (in Planck area units). This result holds in any dimensions $D \geq 3$. In the Reissner-Nordsrom (massive-charged) and Kerr-Newman black hole case (massive-rotating-charged) we show that the Euclidean action in a bulk domain bounded by the inner and outer horizons is the same as the black hole entropy. When one smears out the point-mass and point-charge delta function distributions by a Gaussian distribution, the areaentropy relation is modified. We postulate why these modifications should furnish the logarithmic corrections (and higher inverse powers of the area) to the entropy of these smeared Black Holes. To finalize, we analyse the Bars-Witten stringy black hole in $1+1 \mathrm{dim}$ and its relation to the maximal acceleration principle in phase spaces and Finsler geometries.
\end{abstract}

Keywords : General Relativity; Black Holes; Strings. PACS : 04.60.-m, 04.65.+e, 11.15.-q, 11.30.Ly 


\section{The Difference between a Point Mass Source and the Vacuum Solutions}

We begin by writing down the class of static spherically symmetric (SSS) vacuum solutions of Einstein's equations [1] studied by [5] given by a in finite family of solutions parametrized by a family of admissible radial functions $R(r)$ ( in $c=1$ units )

$$
(d s)^{2}=\left(1-\frac{2 G M}{R}\right)(d t)^{2}-\left(1-\frac{2 G M}{R}\right)^{-1}(d R)^{2}-R^{2}(r)(d \Omega)^{2} .
$$

where the solid angle infinitesimal element is

$$
(d \Omega)^{2}=(d \phi)^{2}+\sin ^{2}(\phi)(d \theta)^{2}
$$

This expression of the metric in terms of the radial function $R(r)$ ( a radial gauge ) does not violate Birkoff's theorem since the metric (1.1, 1.2 ) expressed in terms of the radial function $R(r)$ has exactly the same functional form as that required by Birkoff's theorem and $0 \leq r \leq \infty$. In this work we will solve the SSS solutions when a point mass delta function source is present at the location $r=0$. Notice that the vacuum SSS solutions of Einstein's equations, with and without a cosmological constant, do not determine the form of the radial function $R(r)$. In [34] we were able to show why the cosmological constant is not zero and why it is so tiny based on a judicious choice of the radial function. A Weyl geometric extension of the Jordan-Brans-Dicke scalar-tensor theory gravity [34] furnished also the correct value of the vacuum energy density. In the appendix we construct the Schwarzcshild-like solutions in any dimensions $D>3$ and show that the radial function $R(r)$ is completely arbitrary [45].

There are two interesting cases to study based on the boundary conditions obeyed by $R(r)$ : ( $\mathrm{i}$ ) the Hilbert textbook ( black hole ) solution [4] when $R(r)=r$ obeying $R(r=0)=0, R(r \rightarrow \infty) \rightarrow r$. And : ( ii ) the Abrams-Schwarzschild radial gauge based on choosing the cutoff $R(r=0)=2 G M$ such that $g_{t t}(r=0)=0$ which apparently seems to "eliminate" the horizon and $R(r \rightarrow \infty) \rightarrow r$. This was the original solution of 1916 found by Schwarzschild. However, the choice $R(r=0)=2 G M$ has a serious flaw and is : How is it possible for a point-mass at $r=0$ to have a non-zero area $4 \pi(2 G M)^{2}$ and a zero volume simultaneously? so it seems that one is forced to choose the Hilbert gauge $R(r=0)=0$. Nevertheless we will show how by choosing a judicious choice of $R(r)$ ( not contemplated before to our knowledge), one can cure the flaw and have the correct boundary condition $R(r=0)=0$ while displacing the horizon from $r=2 G M$ to a location arbitrarily close to $r=0$ as one desires, $r_{h} \rightarrow 0$, and where stringy geometry and Quantum Gravitational effects begin to take place. Many authors [5], [6], [7], [8], [9], [13], [10], among many others, have explored the gauge choice obeying $R(r=0)=2 G M$, after Brillouin [3] , Schwarzschild [2] found that possibility long ago. Unfortunately the solution to this serious problem was never found.

In this work we will propose a very straightforward solution to this cut-off problem by choosing a radial gauge function like $R(r)=r+2 G M \Theta(r)$, where the Heaviside Step 
function is defined $\Theta(r)=1$ when $r>0, \Theta(r)=-1$ when $r<0$ and $\Theta(r=0)=0$ (the arithmetic mean of the values at $r>0$ and $r<0$ ). When $R \sim r$ for $r>>2 G M$ and one recovers the correct Newtonian limit in the asymptotic regime. It is now, via the Heaviside step function, that we may maintain the correct behaviour $R(r=0)=0$, when $r=0$, consistent with our intuitive notion that the spatial area and spatial volume of a point $r=0$ has to be zero. ${ }^{1}$

Since $r= \pm \sqrt{x^{2}+y^{2}+z^{2}}$, a negative $r$ branch is mathematically possible and is compatible with the double covering inherent in the Fronsdal-Kruskal-Szekeres [12] analytical continuation in terms of the $u, v$ coordinates. Each point of spacetime inside $r<2 G M$ is represented twice ( black hole and white hole picture ). However there is a fundamental difference (besides others) with the Fronsdal-Kruskal-Szekeres extension into the interior of $r=2 G M$, their metric description is no longer static in $r<2 G M$, whereas in our case the metric is static for all values of $r$.

A rigorous correct treatment of point mass distributions has been provided based on Colombeau's [14] theory of nonlinear distributions, generalized functions and nonlinear calculus. This permits the proper multiplication of distributions since the old Schwarz theory of linear distributions is invalid in nonlinear theories like General Relativity. Colombeau's nonlinear distributional geometry supersedes the no-go results of Geroch and Traschen [16] stating that there is no proper framework to study distributions of matter of co-dimensions higher than two (neither points nor strings in $D=4$ ) in General Relativity. Colombeau's theory of Nonlinear Distributions (and Nonstandard Analysis) is the proper way to deal with point-mass sources in nonlinear theories like Gravity and where one may rigorously solve the problem without having to introduce a boundary of spacetime at $r=0$.

Due to the essential technical subtlety in order to generate $\delta(r)$ terms in the right hand side of Einstein's equations, one must replace everywhere $r \rightarrow|r|$ as required when pointmass sources are inserted. The Newtonian gravitational potential due to a point-mass source at $r=0$ is given by $-G_{N} M /|r|$ and is consistent with Poisson's law which states that the Laplacian of the Newtonian potential $-G M /|r|=4 \pi G \rho$ corresponding to a mass distribution $\rho=\left(M / 4 \pi r^{2}\right) \delta(r)$. However, the Laplacian in spherical coordinates of $(1 / r)$ is zero. For this reason, there is a fundamental difference in dealing with expressions involving absolute values $|r|$ like $1 /|r|$ from those which depend on $r$ like $1 / r$ [11]. Had one not use the modulus $|r|$ in the expression for the metric components $g_{t t}=1-2 G M /|r|$ one will not generate the desired $\delta(r)$ terms in the right hand side of Einstein's equations $\mathcal{R}_{\mu \nu}-\frac{1}{2} g_{\mu \nu} \mathcal{R}=8 \pi G T_{\mu \nu} \neq 0$. Instead, one would get an expression identically equal to zero (consistent with the vacuum solutions in the absence of matter) instead of the $\delta(r)$ terms [35]. Section 3 will be devoted to the study of metrics of the form $g_{\mu \nu}(|r|)$ and $g_{\mu \nu}(|R(r)|)$.

Active diffs must not be confused with passive diffs. One defines an active diffs by

\footnotetext{
${ }^{1}$ We thank Michael Ibison for pointing out the importance of the Heaviside step function and the use of the modulus $|r|$ to account for point mass sources at $r=0$. If one wishes to be strictly rigorous one may write the radial function as $R(r)=r+2 G|M| \Theta(r)$ to ensure that $R(r<0)=-R(r>0)<0$ and that solutions with $r<0, M>0$ have a one-to-one correspondence to the solutions with $r>0, M<0$ ("white hole" ) because $|-M|=|M|$.
} 
mapping $r \rightarrow R(r)$ such that the metric $g_{\mu \nu}[R(r)]$ is diffeomorphic to $g_{\mu \nu}(r)$ if, and only if, the $R(r)$ is a smooth and invertible mapping. However, in order to recover the field due to a point mass delta function source at $r=0$ one must use the modulus function $|r|$ instead of $r$. Since the derivative of the function $|r|$ has a discontinuity at $r=0$, the right and left derivatives are \pm 1 respectively, the second derivative yields a $\delta(r)$ term, the function $R(r)=|r|$ strictly speaking is not smooth in all of the points in a domain enclosing the singularity $r=0$. Consequently, the metric $g_{\mu \nu}(|r|)$ is not diffeomorphic to the Hilbert textbook metric $g_{\mu \nu}(r)$. It is shown in section 3 that the former leads to a scalar curvature $\mathcal{R}=-2 G M \delta(r) / r^{2}$, due to $\delta(-r)=\delta(r)=\delta(|r|)$ and $|r|^{2}=r^{2}$; whereas the latter textbook metric yields an identically zero expression for the Ricci tensor and scalar curvature $\mathcal{R}_{\mu \nu}=\mathcal{R}=0$.

The same reasoning applies to the metric $g_{\mu \nu}(|R(r)|)$ when $|R(r)|=|r+2 G M \Theta(r)|$ and which is not diffeomorphic to the Hilbert textbook metric $g_{\mu \nu}(r)$ nor to the metric $g_{\mu \nu}(|r|)$ due to the fact that the step function $\Theta(r)$ is discontinuous at $r=0$. We will explain why the location of the horizon at $r=2 G M$ is gauge artifact in the sense that it can be displaced to the location $r=0$, in the radial gauge $R(r)=r+2 G M \Theta(r)$, where $\Theta$ is the Heaviside step function. The metric is smooth and differentiable for all $r>0$ and one will have $\mathcal{R}_{\mu \nu}=\mathcal{R}=0$ ( in the region $r>0$ empty of matter and radiation). The metric $g_{\mu \nu}[|R(r)|]$ is discontinuous only at the location of the point mass singularity $r=0$ whose world-line which may be thought of as the boundary of spacetime or transition region to the white hole solution. The scalar curvature is infinite at $r=0$ due to the delta function point mass source at $r=0$, it jumps from zero to infinity at $r=0$.

To sum up, by using $|R(r)|=|r+2 G M \Theta(r)|$, we will have a globally static metric associated with a point mass source at $r=0$ and $g_{\mu \nu}[|R(r)|]$ is not diffeomorphic to the Hilbert textbook metric $g_{\mu \nu}(r)$. The latter is not globally static as FronsdalKruskal-Szekeres have shown after performing the analytical continuation into the region $r<2 G M$ using the $u(r, t), v(r, t)$ coordinates. In such case there is a space-like singularity at $r=0$; it is tachyonic like. Whereas in our case we have a globally static metric with a discontinuity of the metric $g_{\mu \nu}(|R(r)|)$ at the location of the displaced horizon and singularity given by $r=0$. Therefore, because $R(r)=\epsilon+2 G M$, when $r=\epsilon>0$, the horizon to be can the be displaced from $r=2 G M$ to a location as arbitrarily close to $r=0$ as desired $r_{\text {Horizon }} \rightarrow 0$. To be more precise, the horizon occurs at $r=0^{+}$and at $r=0$ one hits the singularity due to the discontinuity of the metric.

Since the notion of distance and separation of spacetime points only has meaning when it is referred to a gravitational field (metric) [18], when one has a metric of the form $g_{\mu \nu}[(R(r)]$, it means that after performing the mapping from $r$ to $R(r)$ in the spacetime manifold $\mathcal{M}$, a void (hole) surrounding $R=r=0$ forms; i.e. a void in the region $0<R<2 G M$ with the singularity remaining at the center $r=0=R(r=0)=0$ and a ring extending from $R=2 G M$ to $R=r=\infty$ ( when $M=$ finite). In the $r$-coordinates picture there is a discontinuity of the metric (and scalar curvature) at $r=0$, the location of the point mass source. Because this is an infinitely compact source there is nothing wrong with having a discontinuity of the metric at $r=0$. In the $R$-coordinate picture, due to the correct condition $R(r=0)=0$ consistent with the fact that a point must have 
zero area (since $\Theta(r=0)=0$ ), one can interpret the discontinuity of the metric as if the region of $0<R<2 G M$ were eliminated from the spacetime manifold to make the surface at $R=2 G M$ a boundary of the spacetime while leaving the singularity at $r=0$ behind.

The Physical reason behind the notion of a spacetime "void" near space-like singularities can be also be inferred from many articles [17] related to the de-emergence of spacetime near spacelike cosmological singularities based on Hyperbolic Kac Moody algebras in $\mathrm{M}$ theory and Cosmological billiards in General Relativity. This spacetime "void" has very deep and profound consequences, in particular, related to the holographic principle where boundary degrees of freedom encode information about the bulk. Roughly speaking, due to the infinite mass density of the point-mass located at $r=0$, its gravitational field will rip and tear apart the fabric of spacetime in its neighborhood and create a "void" in spacetime in the region $0<R<2 G M$. Thus, instead of a Black Hole, we really have a void bulk region $0<R<2 G M$ empty of spacetime while leaving the point-mass singularity at $r=0=R(r=0)=0$ behind.

Having explained why the field of a point mass source at $r=0$ is not given by the Hilbert textbook solution $g_{\mu \nu}(r)$, but instead it is given by non-diffeomorphic solutions of the form $g_{\mu \nu}(|r|)$ and $g_{\mu \nu}(|R(r)|)$, when $R(r)=r+2 G M \Theta(r)$, to be elaborated further in section 3 , we proceed in section 2 with a regularization procedure of the point mass delta function source via a smeared Gaussian mass density and prove why the Euclidean gravitational action involving an Euclidean thermal time is precisely equal to the Entropy. Furthermore, we show that the Euclidean action in a bulk domain bounded by the inner and outer horizons is the same as the Black Hole entropy in the Reissner-Nordsrom (massive-charged) and Kerr-Newman Black Hole case (massive-rotating-charged).

In section 4 another potential solution of the problem of introducing the unacceptable cut-off $R(r=0)=2 G M$ is proposed [45] based on the stringy nature of a "point" mass. A string world-sheet has an area but a zero volume. The Bars-Witten stringy black hole solution in $1+1$-dim [46], [47] with a null horizon at $r=0$ can be embedded into the $3+1$-dim solutions of the form (1.1) involving a very specific radial function (related to the tortoise radial coordinate ) and obeying $R(r=0)=2 G M$, after a conformal rescaling of the metric is performed to ensure that $(d s)^{2} \rightarrow 0$ at $r=0$.

The hallmark of being able to embed the Bars-Witten stringy black hole in $1+1$ into our solutions in $3+1(2+2)$ dimensions [45] is that it has a null horizon at $r=0$ but the singularities lie in the complex plane $r=2 G M(0+i N \pi / 2), N=$ odd. One can complexify the Schwarzschild problem by working with Complex Gravity such that one can have a path from $r=\infty$ to $r=-\infty$ by starting to move along the real axis $r=\rho+i \epsilon$, approach the null horizon $r=0$ at $r=\epsilon+i \epsilon$ to continue upwards along the vertical axis, surround the singularity at $r=2 G M(0+i \pi / 2)$, in a counter-clockwise fashion , move downwards along the vertical axis to $r=-\epsilon+i \epsilon$ and then continue to the $r=-\rho+i \epsilon$ region; i.e. in complex gravity we may be able to tunnel from $r>0$ to $r<0$ through the null horizon at $r=0$ of the Bars-Witten stringy black hole circumventing the singularity at $r=2 G M(0+i \pi / 2)$.

To finalize this section let us outline the most relevant features in order to dispel any misconceptions about our solutions :

- In sections $\mathbf{1}, \mathbf{2}, \mathbf{3}$ it is shown very clearly why the solutions based on the modulus 
function $|r|$ and $|R|=|r+2 G M \Theta(r)|$ are not diffeomorphic to the Hilbert text-book solution; i.e our solutions are not obtained by a change of coordinates (radial reparametrization) of the text-book solution because $r$ and $|r|$ are different functions. $R(r)$ and $|R(r)|$ are different functions as well.

- The solutions described in this work do not have the same expression for the scalar curvature as the text-book solution. Our solutions correspond to an expression for the scalar curvature involving the delta function solution like $\mathcal{R}=-(2 G M \delta(r)) / r^{2}$ as explained in section 3 and which is not the same expression as the identically zero $\mathcal{R}=0$ solution of the text-book solution.

- Our solutions are not obtained by gluing solutions with $M<0$ into the region $r>2 G|M|$. The mass parameter $M>0$. If one wishes to be strictly rigorous one may write the radial function as $R(r)=r+2 G|M| \Theta(r)$ to ensure that $R(r<0)=$ $-R(r>0)<0$ and that solutions with $r<0, M>0$ have a one-to-one correspondence to the solutions with $r>0, M<0$ because $|-M|=|M|$. The latter $M<0$ repulsive gravity regime is what it is called a "white" hole.

- It is shown explicitly in the Appendix that when one plugs eq-(A.12) directly into eq(A.10) and despite that the derivatives $\frac{d R}{d r}=1+2 G|M| \delta(r)$ and $\left(d^{2} R / d r^{2}\right)=2 G|M| \delta^{\prime}(r)$ are singular at $r=0$, there is an exact and precise cancellation of these singular derivatives (and the ordinary derivatives of any radial function $R(r)$ ) in eq-(A.10) ; i.e. the latter eq-(A.10) is satisfied for any radial function, irrespective if it has singular derivatives at $r=0$ or not, for the solutions given by eq-(A.12). Speaking of singular derivatives, it is well known that the Jacobian from the Fronsdal-Kruskal-Szekeres coordinates $u, v$ to the $r, t$ coordinates is singular at the horizon $r=2 G M$.

- Our solutions have a discontinuity of the metric at $r=0$ where the magnitude of the $g_{t t}$ component jumps from 0 to $\infty$ at $r=0$ in the same fashion that the scalar curvature jumps from 0 to $\infty$ at $r=0$. Such discontinuity of the metric at $r=0$ is due to the discontinuity of the radial function given by $R(r=0)=0, R\left(r=0^{+}\right)=2 G M$.

- Having $R(r=0)=0$ and $R\left(r=0^{+}\right)=2 G M$, our solutions near the singularity can be represented by the right and left regions (quadrants) of the Rindler-wedge formed by the straight (null) lines $r=0^{+}, t=+\infty$ and $r=0^{+}, t=-\infty$ at $+45,-45$ degrees respectively. These (null) lines should be compared with the (null) lines $r=2 G M, t= \pm \infty$ corresponding to the text-book solution after performing the Fronsdal-Kruskal-Szekeres change of coordinates

$$
\begin{gathered}
u=\left(\frac{r}{2 G M}-1\right)^{\frac{1}{2}} e^{r / 4 G M} \cosh \left(\frac{t}{4 G M}\right), \quad v=\left(\frac{r}{2 G M}-1\right)^{\frac{1}{2}} e^{r / 4 G M} \sinh \left(\frac{t}{4 G M}\right) \Rightarrow \\
u^{2}-v^{2}=\left(\frac{r}{2 G M}-1\right) e^{r / 2 G M} .
\end{gathered}
$$

leading to a metric

$$
d s^{2}=\frac{4(2 G M)^{3}}{r} e^{-r / 2 G M}\left(d v^{2}-d u^{2}\right)-r^{2}(d \Omega)^{2} .
$$

In our case we must replace $r \rightarrow R$ in eqs- $(1.3,1.4)$ 


$$
\begin{gathered}
u=\left(\frac{R}{2 G M}-1\right)^{\frac{1}{2}} e^{R / 4 G M} \cosh \left(\frac{t}{4 G M}\right), \quad v=\left(\frac{R}{2 G M}-1\right)^{\frac{1}{2}} e^{R / 4 G M} \sinh \left(\frac{t}{4 G M}\right) \Rightarrow \\
u^{2}-v^{2}=\left(\frac{R}{2 G M}-1\right) e^{R / 2 G M}
\end{gathered}
$$

leading to a metric

$$
d s^{2}=\frac{4(2 G M)^{3}}{R} e^{-R / 2 G M}\left(d v^{2}-d u^{2}\right)-R^{2}(d \Omega)^{2} .
$$

such that an incoming photon, starting at point $P$ in the right region (quadrant) of the Rindler wedge, moves upwards parallel to the -45 degrees null-line and reaches the nullline branch given by $r=0^{+}, R\left(r=0^{+}\right)=2 G M$ and $t=\infty$, at point $P^{\prime}$. Then it "tunnels" through the spacetime void and reaches the spacelike singularity $r=0, R(r=0)=0$ at point $P^{\prime \prime}$ whose value of $t\left(P^{\prime \prime}\right)$ is finite. This "tunneling" behaviour from $P^{\prime}$ to $P^{\prime \prime}$ is a direct consequence of the discontinuity of the metric at $r=0$ resuting in a separation between the points $P^{\prime}=\left(r=0^{+}, t=\infty\right)$ and $P^{\prime \prime}=(r=0, t=$ finite $)$.

Similar behaviour occurs for an infalling timelike path starting at $Q$ : it reaches the null-line branch given by $r=0^{+}, R\left(r=0^{+}\right)=2 G M$ and $t=\infty$ at point $Q^{\prime}$. Then it "tunnels" through the spacetime void (due to the discontinuity of the metric at $r=0$ ) reaching the spacelike singularity $r=0, R(r=0)=0$ at point $Q^{\prime \prime}$ whose value of $t\left(Q^{\prime \prime}\right)$ is finite.

In essence, the singularity $r=0, R(r=0)=2 G M$ has been spliced-off from the rest of spacetime by carving out the future and past regions (quadrants) of the Rindler wedge (creating a spacetime void) leaving only the right and left regions (quadrants) bounded by the null lines $r=0^{+}, R\left(r=0^{+}\right)=2 G M$ at $t= \pm \infty$. The fact that we end up only with the left and right regions of the Rindler wedge might have some relationship to the factor of two discrepancy of the Hawking radiation temperature which appears when working with the left-right versus the future-right regions of the Rindler wedge [19].

- Due to the discontinuity of the metric at $r=0$, the location $r=0, R(r=0)=0$ corresponds to a spacelike singularity since $g_{t t}(r=0)=-\infty<0$ : it changes sign. Whereas $g_{r r}(r=0)=0$ because the quantity $r(1+2 G M \delta(r))^{2}=0$ when $r=0$, due to the fact that it is an odd function of $r$ so the latter expression vanishes at $r=0$. Therefore, since $g_{t t}(r=0)=-\infty<0$ has changed sign, it is now spacelike, we must emphasize that no violation of the cosmic censorship conjecture occurs! (that rules out timelike singularities). 


\section{The Euclidean Gravitational Action as Entropy}

\subsection{The Gaussian Distribution as a smeared delta function source and Wave-Particle Duality}

Our aim is to solve the field equations with a delta function point mass source at $r=0$ in $D=3+1$ dimensions. There are two ways of solving this problem. In this section we will smear the point mass delta function distribution using a Gaussian of finite width and calculate the metric, curvature, and stress energy tensor. At the end of the calculations, when one goes back and computes the Einstein-Hilbert action, and takes the $\sigma \rightarrow 0$ limit, one will get the same answer as if one had started with a pure delta function point mass source as shown in this section. This is a consequence of the semi-classical properties inherent in the Gaussian matter distribution and associated with the wave-particle duality in QM; namely, the mass and charge density distributions ( in the case of EM interactions) are just the square of the Gaussian wave function amplitude (times a mass and charge factor) as shown by [21].

Delta-function point sources for general-relativisitic gravity in $1+1$ dimensions yields a rich variety of solutions. Exact solution for 2 point sources on a line, 3 point sources on a line and $N$ point sources on a circle have been found. For 3 point sources the system is chaotic and is a simple model where to study relativistic chaos [20] besides the Kasner-Misner mixmaster chaotic cosmological models. Before doing so, we shall model the mass distribution by a smeared delta function $\rho$ [22], by starting with the following field equations associated with the signature $(+,-,-,-)$

$$
G_{00}=\mathcal{R}_{00}-\frac{1}{2} g_{00} \mathcal{R}=8 \pi G_{N} T_{00}=g_{00} 8 \pi G_{N} \rho(r), \quad \mathcal{R}_{i j}-\frac{1}{2} g_{i j} \mathcal{R}=8 \pi G_{N} T_{i j}
$$

where $\rho(r)$ is a smeared delta function given by the Gaussian, $G_{N}$ is the Newtonian coupling constant ${ }^{2}$ and the $T_{i j}$ elements are comprised of a radial and tangential pressures of a self-gravitating anisotropic fluid [22]

$$
\rho(r)=M_{o} \frac{e^{-r^{2} / 4 \sigma^{2}}}{\left(4 \pi \sigma^{2}\right)^{3 / 2}}, \quad p_{r}=-\rho(r), \quad p_{\text {tan }}=p_{\theta}=p_{\phi}=-\rho(r)-\frac{r}{2} \frac{d \rho}{d r} .
$$

The components of the mixed stress energy tensor are $T_{\nu}^{\mu}=\operatorname{diagonal}\left(-\rho(r), p_{r}, p_{\theta}, p_{\phi}\right)$. The radial pressure $p_{r}=-\rho$ is negative pointing towards the center $r=0$ consistent with the self-gravitating picture of the droplet. The radial dependence of the mass distribution is explicitly given in terms of the incomplete Gamma function $\gamma[a, r]$ as

$$
M(r, \sigma)=M_{o} \int_{0}^{r} \frac{e^{-r^{2} / 4 \sigma^{2}}}{\left(4 \pi \sigma^{2}\right)^{3 / 2}} 4 \pi r^{2} d r=\frac{2 M_{o}}{\sqrt{\pi}} \gamma\left[\frac{3}{2}, \frac{r^{2}}{4 \sigma^{2}}\right] .
$$

\footnotetext{
${ }^{2} G_{N}$ is the Newtonian coupling constant that in some places we write as $G$.
} 
In the limit $\sigma^{2} \rightarrow 0$ one recovers the delta function

$$
\lim _{\sigma \rightarrow 0} \frac{e^{-r^{2} / 4 \sigma^{2}}}{\left(4 \pi \sigma^{2}\right)^{3 / 2}} \rightarrow \frac{\delta(r)}{4 \pi r^{2}} .
$$

and the incomplete Gamma function reduces to the ordinary Gamma function $\Gamma\left(\frac{3}{2}\right)=$ $(\sqrt{\pi} / 2)$ and $M(r, \sigma \rightarrow \infty)$ tends to $M_{o}$. The stress energy tensor for a point mass source is given explicitly by the zero-width limit of the Gaussian in the right hand side of eqs-(2.1, $2.2)$, as shown explicitly in eqs-(2.4), (2.9) and ( 2.10).

The line element which solves Einstein's equations in the presence of the smeared delta function $\rho(r)$ distribution can be obtained by a direct application of Birkoff's theorem by evaluating the (variable ) mass $M(r, \sigma)$ enclosed by a radius $r[22]$

$$
(d s)^{2}=\left(1-\frac{2 G_{N} M(r, \sigma)}{r}\right)(d t)^{2}-\left(1-\frac{2 G_{N} M(r, \sigma)}{r}\right)^{-1}(d r)^{2}-r^{2}(d \Omega)^{2} .
$$

In the Appendix we check that the line element (2.5) based on the radial mass distribution $M(r, \sigma)$ given by the incomplete Gamma function solves Einstein's equations (2.1).

The scalar curvature is given by $8 \pi G_{N}$ trace $\left(T_{\mu \nu}\right)$

$$
\mathcal{R}=-8 \pi G_{N} 2 \rho(r)\left[2-\frac{r^{2}}{4 \sigma^{2}}\right]=-8 \pi G_{N} 2 M_{o} \frac{e^{-r^{2} / 4 \sigma^{2}}}{\left(4 \pi \sigma^{2}\right)^{3 / 2}}\left[2-\frac{r^{2}}{4 \sigma^{2}}\right]
$$

At $r=0$ one has $\mathcal{R}(r=0, \sigma)=-4 G_{N} M_{o} / \sqrt{\pi} \sigma^{3}$ and blows up when $\sigma=0$. At $r=\infty, \mathcal{R}=0$

he $\sigma \rightarrow 0$ limit must be taken after, and only after, performing the calculations. For instance, the Einstein-Hilbert action in the domain bounded by $[0, r]$ will contain the incomplete gammas $\gamma\left[\frac{5}{2}, \frac{r^{2}}{\sigma^{2}}\right]$ and $\gamma\left[\frac{3}{2}, \frac{r^{2}}{\sigma^{2}}\right]$

$$
\begin{gathered}
S=-\frac{1}{16 \pi G_{N}} \int \mathcal{R} \sqrt{|\operatorname{det} g|} d^{4} x= \\
\frac{1}{16 \pi G_{N}} \iint_{0}^{r} 8 \pi G_{N} 2 M_{o} \frac{e^{-r^{2} / 4 \sigma^{2}}}{\left(4 \pi \sigma^{2}\right)^{3 / 2}}\left[2-\frac{r^{2}}{4 \sigma^{2}}\right]\left(4 \pi r^{2} d r d t\right)= \\
\frac{2 M_{o}}{\sqrt{\pi}}\left[2 \gamma\left(\frac{3}{2}, \frac{r^{2}}{\sigma^{2}}\right)-\gamma\left(\frac{5}{2}, \frac{r^{2}}{\sigma^{2}}\right)\right] \int d t .
\end{gathered}
$$

In the $\sigma \rightarrow 0$ limit, the incomplete gammas become the ordinary Euler $\Gamma\left[\frac{5}{2}\right], \Gamma\left[\frac{3}{2}\right]$ giving

$$
\frac{2 M_{o}}{\sqrt{\pi}}\left[2 \frac{1}{2} \sqrt{\pi}-\frac{3}{2} \frac{1}{2} \sqrt{\pi}\right] \int d t=\frac{M_{o}}{2} \int d t .
$$

Thus, the limit $\sigma \rightarrow 0$ has the same effect as if one took the upper $r$ limit of the action integral from $r=$ finite all the way to $r \rightarrow \infty$ irrespective of the value assigned to $\sigma$ ( as long as $\sigma \neq \infty$ ) ; ie. we may evaluate the action all over space (as one should) by fixing the value of $\sigma$ and integrating $r$ from 0 to $\infty$. The incomplete gammas will turn into the 
Euler gammas when $r=\infty$ and one arrives at the same answer (2.7b). Whether or not this has a relationship to the holographic principle is worth investigating.

The Euclideanized Einstein-Hilbert action associated with the scalar curvature in the limit $\sigma \rightarrow 0$ is obtained after a compactification of the temporal direction along a circle $S^{1}$ giving an Euclidean time coordinate interval of $2 \pi t_{E}$ and which is defined in terms of the Hawking temperature $T_{H}$ ( in the limit $\sigma \rightarrow 0$ ) and Boltzman constant $k_{B}$ as $2 \pi t_{E}=\left(1 / k_{B} T_{H}\right)=8 \pi G_{N} M_{o}$. The Euclidean action becomes

$$
S_{E}=\left(\frac{M_{o}}{2}\right)\left(2 \pi t_{E}\right)=4 \pi G_{N} M_{o}^{2}=\frac{1}{4} \frac{4 \pi\left(2 G_{N} M_{o}\right)^{2}}{G_{N}}=\frac{\text { Area }}{4 L_{P}^{2}} .
$$

which is the Black Hole Entropy in Planck area units $G_{N}=L_{P}^{2}(\hbar=c=1)$. We will show below in eq- $(2.7 \mathrm{c})$ that the Euclidean action associated with the scalar curvature corresponding to the delta function point mass source given by

$$
\mathcal{R}=-\frac{2 G_{N} M_{o} \delta(r)}{r^{2}} .
$$

yields precisely the same value for the action and entropy $(2.7 \mathrm{c})$ in the limit $\sigma \rightarrow 0$.

After, and only after, having solved Einstein's equations, one may take the $\sigma \rightarrow 0$ limit of those equations and not before. The order in taking this limiting procedure is essential. It is when this limit is properly taken when one recaptures the true solution due to a point mass delta function source $M_{o}$ at $r=0$. Upon performing the $\sigma=0$ limit in the right order we get

$\rho(r, \sigma=0)=-p_{r}=\frac{M_{o} \delta(r)}{4 \pi r^{2}}, p_{\theta}(r, \sigma=0)=p_{\phi}(r, \sigma=0)=-\frac{M_{o} \delta(r)}{4 \pi r^{2}}-\frac{r}{2} \partial_{r}\left(\frac{M_{o} \delta(r)}{4 \pi r^{2}}\right)$.

such

$$
\begin{gathered}
\mathcal{R}(r, \sigma=0)=-8 \pi G_{N} M_{o}\left[\frac{4 \delta(r)}{4 \pi r^{2}}+r \partial_{r}\left(\frac{\delta(r)}{4 \pi r^{2}}\right)\right] \Rightarrow \\
-\frac{1}{16 \pi G_{N}} \iint \mathcal{R}(r, \sigma=0)\left(4 \pi r^{2} d r d t\right)=-\frac{1}{16 \pi G_{N}} \iint \frac{-2 G_{N} M_{o} \delta(r)}{r^{2}}\left(4 \pi r^{2} d r d t\right) .
\end{gathered}
$$

after an integration by parts since at $r=\infty, r \delta(r) \rightarrow 0$. The relation between the action expressed in terms of $\mathcal{R}(r, \sigma=0)$ and the point mass source delta function case is one of the most important results of this work.

At any given value of $\sigma$, the location of the horizon $r_{s}(\sigma)$ is now shifted to a new location dependent now on the Gaussian width $\sigma$ parameter

$$
1-\frac{2 G_{N} M\left(r_{s}, \sigma\right)}{r_{s}}=0 \Rightarrow r_{s}=2 G_{N} M_{o} \frac{2}{\sqrt{\pi}} \gamma\left[\frac{3}{2}, \frac{r_{s}^{2}}{4 \sigma^{2}}\right]
$$

The solution(s) (if any ) of this transcendental equation yields the new location(s) $r_{s}=$ $r_{s}\left(2 G_{N} M_{o}, \sigma\right)$ of the horizon. In the $\sigma \rightarrow 0$ limit, $r_{s} \rightarrow 2 G_{N} M_{o}$ as expected. The authors [22] plotted the function $g_{00}\left(r, 2 G_{N} M_{o} / \sigma\right)$ and found that there are one, two 
and no horizons depending on the values of the ratio $G_{N} M_{o} / \sigma$. The critical value of the mass below which no horizon forms was $G_{N} M_{o} \sim 1.9 \sigma$ ( our notation differs from $[22])$ which corresponds to $r_{s}\left(2 G_{N} M_{o}, \sigma\right) \sim 3 \sigma$. Similar findings have been found in the Renormalization-Group improved Schwarzchild solutions by [44] based on the running flow of the Newtonian constant $G(r)$ with a non-Gaussian ultraviolet fixed point $G(r=0)=0$ ( asymptotic freedom).

Modifications of the standard thermo-dynamical properties of black holes (logarithmic corrections to the black hole entropy ) based on the solutions of (2.5) within the context of Noncommutative geometry and stringy uncertainty relations have been studied by several authors. See [41] and references therein. In [36] a derivation of the logarithmic corrections to the entropy was found based on a generalized p-Loop oscillator in Clifford spaces and an upper limiting Planck temperature was obtained where Black Hole evaporation stops at the Planck scale.

\subsection{The Euclidean-Action-Entropy Relation in the Point Mass Schwarzschild case}

After having shown how to construct SSS solutions of Einstein's equations in the presence of a delta function mass source at $r=0$, as a limiting procedure $\sigma \rightarrow 0$ of a smeareddelta function mass distribution, we can return now to the introduction of a particular $\sigma$-dependent radial gauge, $R(r, \sigma)=r+r_{s}(\sigma) \Theta(r)$ ( notice the presence of $r_{s}(\sigma)$ ) and replace the metric (2.5) by :

$$
(d s)^{2}=\left(1-\frac{2 G_{N} M(R, \sigma)}{R}\right)(d t)^{2}-\left(1-\frac{2 G_{N} M(R, \sigma)}{R}\right)^{-1}(d R)^{2}-R^{2}(r)(d \Omega)^{2} .
$$

with the upshot that $R(r=0)=0$ as required ( the area and volume of the point $r=0$ has to be zero ) and such that the location of the horizon $r_{h} \rightarrow 0$ can be shifted to a location arbitrarily close to $r=0$, since $R\left(r_{h}=0+\epsilon\right)=\epsilon+r_{s} \sim r_{s}$ where $r_{s}$ is the solution ( if any ) to the prior transcendental equation (2.11) depending on the ratio $G_{N} M_{o} / \sigma[22]$. However, when the radial gauge $R(r)=r+r_{s} \Theta(r)$ is chosen, $r_{s}$ is no longer equal to $r_{h} \rightarrow 0$. Once again, to be more precise, the horizon actually never forms at $r=0$ when $\sigma=0$ (one hits the singularity). When $\sigma \neq 0$, there is one, two and no horizons depending on the values of $G_{N} M_{o} / \sigma[22]$. When there is no horizon then $R(r)=r$. When there is one horizon $R(r)=r+r_{s} \Theta(r)$. When there is an outer $r_{s}^{+}$ and inner horizon $r_{s}^{-}$, by choosing $R(r)=r+r_{s}^{-} \Theta(r)$ then $R \rightarrow r_{s}^{-}$as one approaches $r=0+\epsilon$ and $R(r=0)=0$; while $R=r_{s}^{+}$when $r=r_{s}^{+}-r_{s}^{-}$.

At $r=0 \rightarrow R=0$, the mass is $M(R=0)=0$ ( when $\sigma \neq 0$ ). Therefore, the behaviour of the metric component $g_{00}(R=0)=$ limit $_{R \rightarrow 0}\left(1-2 G_{N} M(R) / R\right)$ requires a very careful evaluation due to the $\frac{0}{0}$ ratio of $\frac{M(R)}{R}$ at $R=0$. Using the properties of the incomplete gammas, the ratio $\frac{M(R)}{R} \sim \gamma\left[\frac{3}{2}, \frac{R^{2}}{4 \sigma^{2}}\right] / R$ for very small values of $R$ behaves as $R^{3} / R=R^{2} \rightarrow 0$. Hence, when $\sigma \neq 0$, the metric component $g_{00}(R=0)=1$ in agreement with the diagrams of $[22]$. 
The scalar curvature corresponding to the metric (2.12) is

$\mathcal{R}=-8 \pi G_{N} 2 \rho(R(r), \sigma)\left[2-\frac{R^{2}(r)}{4 \sigma^{2}}\right]=-16 \pi G_{N} M_{o} \frac{e^{-R(r)^{2} / 4 \sigma^{2}}}{\left(4 \pi \sigma^{2}\right)^{3 / 2}}\left[2-\frac{R^{2}(r)}{4 \sigma^{2}}\right]$

in the $\sigma \rightarrow 0$ limit, the scalar curvature contribution to the action may be evaluated once again by simply inserting

$$
\mathcal{R} \rightarrow-\frac{2 G_{N} M_{o} \delta(r)}{R^{2}(d R / d r)}
$$

into the action in the same manner described by eq-(2.10).

Therefore, the Euclideanized Einstein-Hilbert action associated with the scalar curvature delta function is obtained after a compactification of the temporal direction along a circle $S^{1}$ giving an Euclidean time coordinate interval of $2 \pi t_{E}$ and which is defined in terms of the Hawking temperature $T_{H}$ ( when $\sigma \rightarrow 0$ ) and Boltzman constant $k_{B}$ as $2 \pi t_{E}=\left(1 / k_{B} T_{H}\right)=8 \pi G_{N} M_{o}$. The measure of integration is $4 \pi R^{2} d R d t_{E}$, leading to :

$$
\begin{gathered}
S_{E}=-\frac{1}{16 \pi G_{N}} \iint\left(-\frac{2 G_{N} M_{o}}{R^{2}(d R / d r)} \delta(r)\right)\left(4 \pi R^{2} d R d t\right)= \\
-\frac{1}{16 \pi G_{N}} \iint\left(-\frac{2 G_{N} M_{o}}{r^{2}} \delta(r)\right)\left(4 \pi r^{2} d r d t\right)= \\
\frac{4 \pi\left(G_{N} M_{o}\right)^{2}}{L_{\text {Planck }}^{2}}=\frac{4 \pi\left(2 G_{N} M_{o}\right)^{2}}{4 L_{\text {Planck }}^{2}}=\frac{\text { Area }}{4 L_{\text {Planck }}^{2}}= \\
\frac{1}{16 \pi G_{N}} \lim _{\sigma \rightarrow 0} \iint_{0}^{R} 16 \pi G_{N} M_{o} \frac{e^{-R(r)^{2} / 4 \sigma^{2}}}{\left(4 \pi \sigma^{2}\right)^{3 / 2}}\left[2-\frac{R^{2}(r)}{4 \sigma^{2}}\right]\left(4 \pi R^{2} d R d t\right)= \\
\frac{1}{16 \pi G_{N}} \iint_{0}^{\infty} 16 \pi G_{N} M_{o} \frac{e^{-R(r)^{2} / 4 \sigma^{2}}}{\left(4 \pi \sigma^{2}\right)^{3 / 2}}\left[2-\frac{R^{2}(r)}{4 \sigma^{2}}\right]\left(4 \pi R^{2} d R d t\right)
\end{gathered}
$$

when equating $G_{N}=L_{P}^{2}$ and after performing the integration in terms of the incomplete gammas and taking the $\sigma \rightarrow 0$ limit. Once again, the limit $\sigma \rightarrow 0$ has the same effect as if one took the upper $R$ limit of the action integral from $R=$ finite all the way to $R \rightarrow \infty$ ( as one should to define the action ) while keeping $\sigma$ fixed. It is interesting that the Euclidean action ( in $\hbar$ units ) is precisely the same as the black hole entropy in Planck area units. This result holds in any dimensions $D \geq 3$. This is not a numerical coincidence and is deeply related to the thermal nature of Euclidean time; namely, the conserved global charges associated to the Euclidean Einstein-Hilbert action obey a relation that could be interpreted as a thermodynamical equation of state. Furthermore, the action is invariant of the choices of $R(r)$, whether or not it is the Hilbert text book choice $R(r)=r$ or another. The choice of the radial function $R(r)$ amounts to a radial gauge that leaves the action invariant but it does not leave the scalar curvature, nor the measure of integration, invariant. Only the action ( integral of the scalar curvature ) remains invariant. This follows directly from the relations $\mathcal{R}[R(r)]=\mathcal{R}^{\prime}(r) \neq \mathcal{R}(r)$ and $\mathcal{R}(r)=\mathcal{R}^{\prime}\left(r^{\prime}\right)$. 
The physical picture behind the Euclidean action = entropy relation is based on the wave-particle duality in QM where the Gaussian mass density distribution $\rho=M_{o}|\Psi|^{2}$ is proportional to the square of the Gaussian wave amplitude. [21]. One can imagine the point mass delta function source at $r=0$ as being smeared all over spacetime, from $r=0$ to $r=\infty$ by a Gaussian mass density distribution of a variable width $\sigma$. As long as $\sigma \neq \infty$ the answer (2.14) is the same whether one integrates all over space, keeping $\sigma$ fixed, or if one integrates up to a given $r$ and takes the subsequent $\sigma=0$ limit, consistent with the delta function distribution being the zero width limit of a Gaussian. In the delta function case for the scalar curvature, we know that $\mathcal{R}=0, r>0$ thus the contribution to the action is zero for all $r>0$; only the delta function behaviour of the scalar curvature at $r=0$ contributes to the action. A detailed study of the nonzero $\sigma$ modifications of the Hawking temperature, entropy and horizon may be found in [22], [41], [24]. However, as we have seen in the last term of eq-(2.14), when one integrates all over space for any fixed value of $\sigma \neq \infty$ one always gets the same answer in terms of the mass parameter $S_{E}=4 \pi G M_{o}^{2}$, if, and only if, $k_{B} T_{H}=\left(8 \pi G_{N} M_{o}\right)^{-1}$.

If it is only the relationship between $M_{o}$ and the modified horizon $r_{s}=r_{s}\left(2 G_{N} M_{o}, \sigma\right)$ that changes when $\sigma \neq 0$, the immediate question is what functional relation between the area $\left(4 \pi r_{s}^{2}\right)$ and entropy one would find if we restricted the domain of integration from $r=0$ to $r_{s}$ for fixed values of $\sigma \neq 0$ ? If one views the Hawking emission of particles as a tunneling phenomenon through the horizon [25], when there are two horizon solutions to the transcendental equation (2.11) one may compute the Euclidean Einstein-Hilbert action in the bounded domain determined by the outer and inner horizons $r_{ \pm}(\sigma)$ defined by :

$$
S_{E}=\frac{1}{16 \pi G_{N}} \int_{r_{-}(\sigma)}^{r_{+}(\sigma)} 16 \pi G_{N} M_{o} \frac{e^{-r^{2} / 4 \sigma^{2}}}{\left(4 \pi \sigma^{2}\right)^{3 / 2}}\left[2-\frac{r^{2}}{4 \sigma^{2}}\right]\left(4 \pi r^{2} d r\right) \int_{0}^{1 / k_{B} T} d t_{E}
$$

The Euclidean time interval, the thermal time interval, $2 \pi t_{E}=1 / k_{B} T_{H}(\sigma)$ is given in terms of the inverse of the deformed Hawking temperature ( setting the Boltzman constant $k_{B}=1$ ) by the formula [22]

$$
T_{H}(\sigma)=\frac{1}{4 \pi} \frac{d g_{t t}}{d r}\left(r=r_{+}(\sigma)\right)=\frac{1}{4 \pi r_{+}(\sigma)}\left[1-\frac{r_{+}^{3}(\sigma)}{4 \sigma^{3}} \frac{e^{-r_{+}^{2}(\sigma) / 4 \sigma^{2}}}{\gamma\left[\frac{3}{2} ; \frac{r_{+}^{2}(\sigma)}{4 \sigma^{2}}\right]}\right] .
$$

Hence the inverse temperature ( the Euclidean time interval of integration ) is

$$
\frac{1}{T_{H}(\sigma)}=4 \pi r_{+}(\sigma)\left[1-\frac{r_{+}^{3}(\sigma)}{4 \sigma^{3}} \frac{e^{-r_{+}^{2}(\sigma) / 4 \sigma^{2}}}{\gamma\left[\frac{3}{2} ; \frac{r_{+}^{2}(\sigma)}{4 \sigma^{2}}\right]}\right]^{-1}
$$

and the Euclidean action bounded by the outer and inner horizons $r_{ \pm}(\sigma)$ is

$$
S_{E}=\frac{1}{16 \pi G_{N}} \int_{r_{-}(\sigma)}^{r_{+}(\sigma)} 16 \pi G_{N} M_{o} \frac{e^{-r^{2} / 4 \sigma^{2}}}{\left(4 \pi \sigma^{2}\right)^{3 / 2}}\left[2-\frac{r^{2}}{4 \sigma^{2}}\right]\left(4 \pi r^{2} d r\right) \times
$$




$$
4 \pi r_{+}(\sigma)\left[1-\frac{r_{+}^{3}(\sigma)}{4 \sigma^{3}} \frac{e^{-r_{+}^{2}(\sigma) / 4 \sigma^{2}}}{\gamma\left[\frac{3}{2} ; \frac{r_{+}^{2}(\sigma)}{4 \sigma^{2}}\right]}\right]^{-1}
$$

In the $\sigma \rightarrow 0$ limit ( equivalent to the large $M_{o} / \sigma \rightarrow \infty$ limit ) the inner horizon shrinks to zero $r_{-}(\sigma) \rightarrow 0[22]$, the outer horizon $r_{+}(\sigma) \rightarrow 2 G M$ and the deformed Hawking temperature becomes the ordinary one $T_{H}(\sigma) \rightarrow 1 / 8 \pi G M$. The spatial integral of the scalar curvature ( times $1 / 16 \pi G_{N}$ ) in the $\sigma \rightarrow 0$ limit was already evaluated in eq-(2.10) after an integration by parts of the derivatives of the delta function terms, and it yields $M_{o} / 2$ because the domain of integration contains $r=0$. Therefore, in the $\sigma \rightarrow 0$ limit of eq-(2.18) one recovers the usual result of $S_{E}=\frac{1}{4}\left(\right.$ Area $\left./ L_{P}^{2}\right)=\frac{1}{4 G_{N}} 4 \pi\left(2 G_{N} M\right)^{2}=$ $4 \pi G_{N} M^{2}$.

The impending question now is what is the functional relationship between the Euclidean action bounded by the outer and inner horizons of eq-(2.18) and the area of the outer horizon $4 \pi r_{+}(\sigma)^{2}$ ? Due to the fact the the expression for the inner and outer horizons $r_{ \pm}(\sigma)$ is no longer analytic, we postulate that this modified Euclidean actionarea-entropy relation could be expressed in terms of a power series (logarithmic corrections to the entropy) of the form

$$
S[A(\sigma)]=\frac{A(\sigma)}{4 G_{N}}+b(\sigma) \log \frac{A(\sigma)}{4 G_{N}}+\sum_{n=0}^{n=\infty} a_{n}(\sigma)\left(\frac{A(\sigma)}{4 G_{N}}\right)^{-n}, \quad A(\sigma)=4 \pi r_{+}(\sigma)^{2}
$$

where the coefficients $b(\sigma)$ and $a_{n}(\sigma)$ are explicitly $\sigma$-dependent. In the $\sigma \rightarrow 0$ limit one recovers the standard action-area-entropy relation in natural units $\hbar=c=k_{B}=1$ $S[A(\sigma=0)]=A(\sigma=0) / 4 G_{N}=4 \pi\left(2 G_{N} M\right)^{2} / 4 G_{N}=4 \pi G_{N} M^{2}$.

Another way to obtain the corrections to the entropy-area law is by integrating the first law of black hole thermo-dynamics

$$
T d S=d M \Rightarrow S=\int \frac{d M}{T(M, \sigma)}
$$

where the expression $T(M, \sigma)$ is not analytical and is implicitly given in terms of the outer horizon solutions $r_{+}(\sigma ; M)$ given by eqs- $(2.11,2.16)$. The authors [41] have obtained the corrections to the area-entropy relation by recurring to the minimal length stringy uncertainty relations by postulating a mass-temperature relation of the form

$$
(\Delta x)(\Delta p) \geq \frac{1}{2}\left(1+a^{2} G(\Delta p)^{2}\right) \Rightarrow(2 G M)\left(k_{B} T\right) \sim \frac{1}{2}\left(1+\alpha^{2} G\left(k_{B} T\right)^{2}\right) .
$$

that allows to find the solve for $T=T(M)$ and to integrate (2.19b). For further details we refer to [41].

The most salient feature of eq-(2.14), besides the action-entropy relation, can be inferred by looking at the second term of (1.16). By replacing $r \rightarrow-r, t_{E} \rightarrow-t_{E} \Rightarrow T \rightarrow$ $-T$ and because $\delta(r)=\delta(-r)$, one leaves the action, the entropy, invariant. Therefore, negative temperatures ( but positive entropy) are inherently associated with the repulsive gravity white-hole picture in the region $r<0$. The concept of anti-entropy 
is a very different story that has been explored by mathematical biologists and deserves further investigation. Even further, by working with Complex Gravity, the complex time $\tau=t+i \beta$ has for imaginary component $\beta=1 / k_{B} T$, and allows to extend ordinary QM to Complex QM where the Hamiltonians are no longer Hermitians which is what one expects due to the irrever sibility nature of a complex temporal evolution of the complex Hamiltonian. A complex time associated with Complex Gravity is where one can more rigorously implement the notion of $S$-duality in linearized Gravity [49] to the full nonlinear theory.

The action - entropy connection has been obtained from a different argument, for example, by Padmanabhan [32] by showing how it is the surface term added to the action which is related to the entropy, interpreting the horizon as a boundary of spacetime. The surface term is given in terms of the trace of the extrinsic curvature of the boundary. The surface term in the action is directly related to the observer-dependent-horizon entropy, such that its variation, when the horizon is moved infinitesimally, is equivalent to the change of entropy $d \mathcal{S}$ due to the virtual work. The variational principle is equivalent to the thermodynamic identity $T d \mathcal{S}=d E+p d V$ due to the variation of the matter terms in the right hand side. A bulk and boundary stress energy tensors are required to capture the Hawking thermal radiation flux seen by an asymptotic observer at infinity as the black hole evaporates.

Finding dynamical solutions $M(r, t, \sigma)$ are the subject of another investigation. The author [37] by using the principle of invariance of the 4-dim world-volume has found that the gravitational mass of the neutral mass-point tends to zero, after an eternal gravitational collapse of a star has shed off all of its mass, by radiating away its available mass energy ( to infinity ). Notice, however, that the measure $4 \pi R^{2} d R d t$ is not invariant, when we change the radial gauges, for example, from $R(r)=r+r_{s}(\sigma) \Theta(r)$ to another $R(r)=\left[r^{2}+r_{s}^{2}(\sigma) \Theta(r)\right]^{1 / 2}$. Thus one can bypass the results of [37] that relied on the invariance of the 4-dim measure. Furthermore, the argument of [37] relies on having a finite value of the bare mass which may not be the case. Once again, what remains invariant is the action (2.14) as a whole, not the measure, nor the scalar curvature $\mathcal{R}$. We have shown that the gravitational field of a point mass delta function source is a mathematically sound problem that has a solution for $M \neq 0$. Another problem that needs to be revisited is the gravitational collapse of a compact star taking into account the freedom of choosing radial gauges involving the step function that have not been explored before.

Naturally, Quantum Gravitational effects become very relevant at small (Planck) scales. The ultra-violet cutoff $R(r=0)=2 G_{N} M_{o}$ is compatible with the exact Nonperturbative Renormalization Group flow of the Newtonian coupling $G=G(r)$ (that has a non-Gaussian ultraviolet fixed point $G(r=0)=0$ ) in Quantum Einstein Gravity [44] based on Weinberg's Asymptotic Safety scenario. The presence of an ultra-violet cutoff $R=2 G_{N} M_{o}$ originates from the mere presence of matter and permits to relate $g_{t t}=1-2 G_{N} M_{o} / R(r)$ to $g_{t t}=1-2 G(r) M(r) / r$ such that $g_{t t}(r=0)=0$ and which is compatible with the ultra-violet cutoff of the radial function $R(r=0)=2 G_{N} M_{o}$. $G_{N}$ is the value of the Newtonian coupling in the deep infrared and $M=M_{o}$ is the Kepler mass as seen by an observer at asymptotic infinity. The non-perturbative exact Renormaliza- 
tion Group program for Quantum Einstein Gravity helps to determine the choice $R(r)$ uniquely $R=r+2 G_{N} M_{o}$ from the infinite family of plausible radial functions $R(r)$.

When $r=0$ a careful analysis yields $M(r \rightarrow 0) \sim \frac{1}{2 G_{N} M_{o}}$ therefore, the running mass parameter at $r=0, M(r=0) \sim 1 / R(r=0)=1 /\left(2 G_{N} M_{o}\right)$ is finite instead of being infinite. The running mass at $r=0$ has a cutoff given by the inverse of the ultraviolet cutoff $R(r=0)=2 G_{N} M_{o}$ ( up to a numerical constant ). If one includes the step function in $R(r)=r+2 G_{N} M \Theta(r)$ there is no cutoff $R(r=0)=0$ and the bare mass should be $M(r=0)=\infty$ as expected. When $r \rightarrow \infty$ one has $M(r \rightarrow \infty) \rightarrow M_{o}$ as expected, where $M_{o}$ is the Kepler mass observed by an observer at asymptotic infinity ( deep infrared ). For further details we refer to [43].

\subsection{The Euclidean Action-Entropy Relation in the Charged- Kerr Black holes case}

Let us begin with the Einstein-Maxwell action

$$
\begin{aligned}
S=- & \frac{1}{16 \pi G} \int d^{4} x \sqrt{g} \mathcal{R}+\frac{1}{4 e^{2}} \int d^{4} x \sqrt{g} F_{\mu \nu} F^{\mu \nu}= \\
& -\frac{1}{16 \pi G} \int d^{4} x \sqrt{g}\left[\mathcal{R}-\frac{4 \pi G}{e^{2}} F_{\mu \nu} F^{\mu \nu}\right] .
\end{aligned}
$$

We will calculate the entropy in the special case when the charge $e$ satisfies the condition $4 \pi G=e^{2}$, the charge $e$ has length units. In this particular case the Euclidean action matches the entropy. In the case that $4 \pi G \neq e^{2}$ the Euclidean action is proportional to the black hole entropy. The constant of proportionality is $4 \pi G / e^{2}$.

The charged massive Reissner-Nordstrom solution has for metric components

$$
g_{t t}=1-\frac{2 G_{N} M}{r}+\frac{e^{2}}{r^{2}}, \quad g_{r r}=-\frac{1}{g_{t t}} .
$$

the angular part is the same $r^{2}(d \Omega)^{2}$. In the point mass and point charge case, we should replace $r \rightarrow|r|$ in order to recover delta function point mass and point charge singularities at $r=0$. In the region $r>0$ the only contribution to the field equations is from the EM field stress-energy tensor. Einstein's equations in the case that $4 \pi G=e^{2}$ are

$$
\begin{gathered}
\frac{\delta S}{\delta g^{\mu \nu}}=0 \Rightarrow \mathcal{R}_{\mu \nu}-\frac{1}{2} g_{\mu \nu} \mathcal{R}=8 \pi G T_{\mu \nu}= \\
-\left[g^{\alpha \beta}\left(F_{\mu \alpha} F_{\nu \beta}+F_{\nu \alpha} F_{\mu \beta}\right)-\frac{1}{2} g_{\mu \nu} F^{\alpha \beta} F_{\alpha \beta}\right], r>0 .
\end{gathered}
$$

In $D=4$ the trace of the stress EM energy tensor is zero consistent with the conformal invariance of the Maxwell action in $D=4$. This results simply follows from a variation under conformal scalings

$$
\text { if } 2 \delta S=-\sqrt{g} T_{\mu \nu} \delta g^{\mu \nu}=-\lambda \sqrt{g} w\left(g^{\mu \nu}\right) T_{\mu \nu} g^{\mu \nu}=0 \Rightarrow T=T_{\mu \nu} g^{\mu \nu}=0 \text {. }
$$


since the Weyl weight $w\left(g^{\mu \nu}\right) \neq 0$. The minus sign of the second term in the r.h.s of $(2.22)$ is due to the variation of the determinant of the metric $g_{\mu \nu}$ resulting from the identities

$$
\begin{gathered}
\sqrt{\operatorname{det} g_{\mu \nu}}=e^{\frac{1}{2} \operatorname{trace} \ln \left(g_{\mu \nu}\right)}=e^{-\frac{1}{2} \operatorname{trace} \ln \left(g^{\mu \nu}\right)}, \quad g^{\mu \nu}=\left(g_{\mu \nu}\right)^{-1} . \\
\delta \sqrt{g}=-\frac{1}{2} \sqrt{g} g_{\mu \nu} \delta g^{\mu \nu}=\frac{1}{2} \sqrt{g} g^{\mu \nu} \delta g_{\mu \nu}, .
\end{gathered}
$$

Therefore, when $r>0$ the point mass terms don't contribute to the stress energy tensor and the relevant term is then the EM part of the action density :

$$
\frac{1}{4 e^{2}} F_{\alpha \beta} F^{\alpha \beta}=\frac{E^{2}(r)}{4 e^{2}}=\frac{1}{4 e^{2}}\left(\frac{e}{r^{2}}\right)^{2}=\frac{1}{16 \pi G} \frac{e^{2}}{r^{4}} \text {, when } 4 \pi G=e^{2} .
$$

the outer and inner horizons of the Reissner-Nordstrom massive charged black hole in the natural units is given by the solutions of the algebraic equation

$$
1-\frac{2 G M}{r}+\frac{e^{2}}{r^{2}}=0 \Rightarrow r_{ \pm}=G M \pm \sqrt{(G M)^{2}-e^{2}} .
$$

From eq-(2.25) we can evaluate the EM part of the action bounded by the outer and inner horizons of the Reissner-Nordstrom massive charged black hole

$$
\frac{1}{16 \pi G} \iint_{r_{-}}^{r_{+}} F_{\mu \nu} F^{\mu \nu}\left(4 \pi r^{2} d r d t\right)
$$

The spatial integral yields

$$
\begin{gathered}
\frac{1}{16 \pi G} \int_{r_{-}}^{r_{+}} \frac{e^{2}}{r^{4}} 4 \pi r^{2} d r= \\
\frac{e^{2}}{4 G}\left[\frac{1}{r_{-}}-\frac{1}{r_{+}}\right]=\frac{e^{2}}{4 G} \frac{r_{+}-r_{-}}{r_{+} r_{-}}=\frac{e^{2}}{4 G} \frac{2 \sqrt{(G M)^{2}-e^{2}}}{e^{2}} .
\end{gathered}
$$

Upon a compactification the Euclidean thermal-time interval is $2 \pi t=1 / k_{B} T$ ( we will set $k_{B}=1$ ). The temperature of the Reissner-Nordstrom Black Hole [38] is

$$
T_{H}=\frac{1}{2 \pi} \frac{\sqrt{(G M)^{2}-e^{2}}}{2(G M)^{2}+2 G M \sqrt{(G M)^{2}-e^{2}}-e^{2}}=\frac{1}{4 \pi} \frac{r_{+}-r_{-}}{r_{+}^{2}} .
$$

The full spatial-temporal integration corresponding to the EM part of the action bounded by the outer and inner horizons of the Reissner-Nordstrom Black Hole is then :

$$
\begin{gathered}
\frac{1}{16 \pi G} \int_{r_{-}}^{r_{+}} \frac{e^{2}}{r^{4}}\left(4 \pi r^{2} d r\right) \int_{0}^{1 / k_{B} T} d t_{E}= \\
{\left[\frac{2 \pi e^{2}}{4 G}\right]\left[\frac{2 \sqrt{(G M)^{2}-e^{2}}}{e^{2}}\right]\left[\frac{2(G M)^{2}+2 G M \sqrt{(G M)^{2}-e^{2}}-e^{2}}{\sqrt{(G M)^{2}-e^{2}}}\right]=}
\end{gathered}
$$




$$
\left[\frac{\pi}{G}\right]\left[G M+\sqrt{(G M)^{2}-e^{2}}\right]^{2}=\frac{\pi r_{+}^{2}}{G}=\frac{4 \pi r_{+}^{2}}{4 G}=\frac{\text { Area }}{4 L_{\text {Planck }}^{2}} .
$$

Therefore, we have shown that when $4 \pi G=e^{2}$, the (Euclideanized) EM part of the action associated with the bulk region bounded by the outer and inner horizons of the Reissner-Nordstrom massive charged black hole is precisely equal to the Black Hole Entropy $4 \pi r_{+}^{2} / 4 G$. The relationship between $G$ and $e$ is reminiscent of what occurs in Kaluza-Klein compactifications from $5 D$ to $4 D$. In Loop Quantum Gravity there is an undetermined Immirizi parameter in the Entropy-Area relation based on the $S U(2)$ spinnetworks calculation.

The Reissner-Nordstrom black hole entropy can be recast as

$$
S_{E}^{R N}=\frac{e^{2}}{4 G}\left[\frac{1}{r_{-}}-\frac{1}{r_{+}}\right] \frac{1}{T_{H}}=\frac{e^{2}}{4 G} \frac{r_{+}-r_{-}}{r_{+} r_{-}} \frac{4 \pi r_{+}^{2}}{r_{+}-r_{-}}=\frac{4 \pi r_{+}^{2}}{4 G}
$$

since $e^{2}=r_{+} r_{-}$resulting from eq-(2.26) and after using eq-(2.29). In the extremal Reissner-Nordstrom black hole case, $G M=e$, the outer and inner horizons coincide $r_{+}=r_{-}=G M$ so the spatial integral of the scalar curvature bounded by a domain of size zero is zero. However since the temperature in this extremal case is also zero, when one computes the Entropy in this case one will get $\frac{0}{0}$ which is undetermined, however due to the exact cancellation of the terms $r_{+}-r_{-}$in the numerator and denominator of eq(2.31) the Entropy value becomes precisely equal to $\left(\right.$ Area/4G) where Area $=4 \pi r_{+}^{2}=$ $4 \pi r_{-}^{2}=4 \pi(G M)^{2}$ for the extremal Reissner-Nordstrom Black hole.

The charged rotating ring solution given by the Kerr-Newman Black Hole has an angular momentum per unit mass $a=\frac{J}{M}$ and the Hawking temperature corresponding to the outer horizon is [38]

$$
\begin{gathered}
T_{H}\left(M, e, a=\frac{J}{M}\right)=\frac{1}{2 \pi} \frac{\sqrt{(G M)^{2}-a^{2}-e^{2}}}{2(G M)^{2}+2 G M \sqrt{\left.(G M)^{2}-a^{2}-e^{2}\right)}-e^{2}}= \\
\frac{1}{4 \pi} \frac{r_{+}-r_{-}}{r_{+}^{2}+a^{2}} .
\end{gathered}
$$

The outer and inner horizons are solutions of the equation :

$$
\Delta=r^{2}-2 G M r+a^{2}+e^{2}=0 \Rightarrow r_{ \pm}=G M \pm \sqrt{(G M)^{2}-a^{2}-e^{2}} .
$$

the Entropy is

$$
S_{E}=\frac{1}{4 G} 4 \pi\left[r_{+}^{2}+a^{2}\right]=\left[\frac{\pi}{G}\right]\left[G M+\sqrt{(G M)^{2}-a^{2}-e^{2}}\right]^{2}=\frac{\text { Area }}{4 L_{\text {Planck }}^{2}} .
$$

As a direct consequence of the temperature relation (2.32) and the rotational-energy density $(1 / 16 \pi G)\left(J / M r^{2}\right)^{2}=(1 / 16 \pi G)\left(a / r^{2}\right)^{2}$ contribution to the energy, the Kerr- 
Newman back-hole entropy can still be re-written as

$S_{E}^{K N}=\frac{e^{2}+a^{2}}{4 G}\left[\frac{1}{r_{-}}-\frac{1}{r_{+}}\right] \frac{1}{T_{H}}=\frac{e^{2}+a^{2}}{4 G} \frac{r_{+}-r_{-}}{r_{+} r_{-}} \frac{4 \pi\left(r_{+}^{2}+a^{2}\right)}{r_{+}-r_{-}}=\frac{4 \pi\left(r_{+}^{2}+a^{2}\right)}{4 G}$.

since $e^{2}+a^{2}=r_{+} r_{-}$resulting from (2.33). Based on the entropy functional forms given by eqs- $(2.31,2.35)$ (in the form energy $\times$ time) of the Reissner-Nordstrom and KerrNewman back-hole entropies, it is warranted to compare these expressions with those corresponding to charged and spinning p-branes. For instance, the charge-mass-spin (angular momentum) relationship of the Kerr-Newman back-hole horizon was re-derived within the context of poly-particle actions in Clifford poly-vector valued spaces in [62].

If one evaluates the bulk integral of the EM part of the action $\frac{1}{4 e^{2}} F_{\mu \nu} F^{\mu \nu}$ in the KerrNewman stationary solution expressed in Boyer-Lindquist coordinates one gets

$$
\begin{gathered}
S_{E}=\frac{1}{4 e^{2}} \int d t \int d \phi \int_{r_{-}}^{r_{+}} d r \int_{-1}^{+1} d(\cos \phi)\left(r^{2}+a^{2} \cos ^{2} \phi\right) \frac{e^{2}}{\left(r^{2}+a^{2} \cos ^{2} \phi\right)^{2}}= \\
\pi\left[\frac{i L i_{2}\left[-\frac{i a}{r}\right]-i L i_{2}\left[\frac{i a}{r}\right]}{2 a}\right]_{r_{-}}^{r_{+}} \frac{1}{T_{H}} .
\end{gathered}
$$

where $L i_{2}$ is the di-logarithm. The poly-logarithm is defined by $L i_{n}(z)=\sum_{k=1} \frac{z^{k}}{k^{n}}$. Hence, it is only to leading order in powers of $\frac{a}{r}$ that one recovers from the integral of eq- $(2.36)$ the Kerr-Newman black hole entropy

$$
\begin{aligned}
& S_{E} \sim \pi\left[\frac{1}{r_{-}}-\frac{1}{r_{+}}\right] \frac{1}{T_{H}}=\frac{\pi\left(r_{+}-r_{-}\right)}{r_{+} r_{-}} \frac{4 \pi\left(r_{+}^{2}+a^{2}\right)}{r_{+}-r_{-}}= \\
& \frac{\pi}{e^{2}+a^{2}} 4 \pi\left(r_{+}^{2}+a^{2}\right)=\frac{4 \pi\left(r_{+}^{2}+a^{2}\right)}{4 G}, \text { when } e^{2}+a^{2}=4 \pi G .
\end{aligned}
$$

with the provision that the condition $e^{2}+a^{2}=4 \pi G$ is obeyed. The Reissner-Nordstrom entropy was recovered in eq-(2.30) when the condition $e^{2}=4 \pi G$ was satisfied. To sum up, the expression

$$
S_{E}=\frac{e^{2}+a^{2}}{4 G}\left[\frac{1}{r_{-}}-\frac{1}{r_{+}}\right] \frac{1}{T_{H}} .
$$

recaptures the Kerr-Newman entropy as well as the Reissner-Nordstrom, Kerr and Schwarzschild entropy when $a^{2}=0, e^{2}=0, e^{2}=a^{2}=0$, respectively.

The first law of thermodynamics relating the change of the internal energy $d U$ with the change of entropy $T d S$ and work $d W$ is :

$$
T d S_{E}-d W=d U \Rightarrow T d S_{E}-J d \Omega-\Phi d Q=d U=d(M-Q \Phi-J \Omega) .
$$

where $\Phi=e / r_{+}$is the electrostatic potential at the outer-horizon, $\Omega$ is the angular velocity of the outer horizon; $J$ is the angular momentum and $M$ is the ADM mass. The first law 
can be interpreted as the relationship among the global charges, parameters $(M, e, J)$ and $T, S$ which is obtained by performing a variation of the Euclidean action resulting from perturbing the location of the inner and outer horizons. Viewing the Hawking radiation and emission of particles as a quantum tunneling that shrinks the size of the horizons [25] is another way of perturbing the value of the Euclidean action. For a thorough discussion of interpreting Einstein's field equations as just a thermo-dynamical equation of state see [26] and Wald's entropy expression related to the global Noether charge of diffs under the Killing vector field which generates the event horizon in the stationary black hole background and which is given by a local geometric density integrated over a space-like section of the horizon [39]. An analysis of non-extremal black hole entropy and $U$ duality in four dimensions was provided by [40].

One could smear the point mass and point charge distributions by a Gaussian of variable width and height and repeat the whole exercise of the previous section. The $\sigma$-modified Reissner-Nordstrom Black hole has a metric given by

$$
g_{t t}=1-\frac{2 G m(r, \sigma)}{r}+\frac{e^{2} f(r, \sigma)}{r^{2}}, \quad g_{r r}=-\frac{1}{g_{t t}} .
$$

where $f(r)$ is determined by solving the coupled Einstein-Maxell equations

$$
\begin{gathered}
\mathcal{R}_{\mu \nu}-\frac{1}{2} g_{\mu \nu} \mathcal{R}=-8 \pi G \frac{1}{2}\left[g^{\alpha \beta} F_{\mu \alpha} F_{\nu \beta}+\mu \leftrightarrow \nu\right. \\
\frac{1}{\sqrt{|g|}} \partial_{\mu}\left[\sqrt{|g|} F^{\mu \nu}\right]=J^{\nu}
\end{gathered}
$$

since the functions are known : $m(r)=\frac{2 m_{o}}{\sqrt{\pi}} \gamma\left[\frac{3}{2}, \frac{r^{2}}{4 \sigma^{2}}\right]$ and a similar expression for the charge $q(r)$ enclosed by $r$ and which furnishes the electrostatic field at any point $E(r)=q(r) / r^{2}$ by a simple application of Gauss law. The authors [23] found that the functional form of $f(r)$ is :

$$
f(r)=\frac{1}{\pi} \gamma\left[\frac{1}{2}, \frac{r^{2}}{4 \sigma^{2}}\right]^{2}-\frac{r}{\sqrt{2} \pi \sigma} \gamma\left[\frac{1}{2}, \frac{r^{2}}{4 \sigma^{2}}\right] .
$$

$f(r)$ has a singularity at $\sigma=0$. If the metric is expressed in terms of the ADM mass $M$

$$
g_{t t}=1-\frac{2 G M(r, \sigma)}{r}+\frac{e^{2} F(r, \sigma)}{r^{2}} .
$$

the new function $F(r)$ obtained by [23]

$$
F(r)=f(r)+\frac{\sqrt{2} r}{\pi \sigma} \gamma\left[\frac{3}{2}, \frac{r^{2}}{4 \sigma^{2}}\right]
$$

$F(r)$ is not singular at $\sigma=0$ due to the cancellation of the $1 / \sigma$ terms when $\sigma=0$ resulting from the limit $\gamma[c, x] \rightarrow$ Euler gammas when $x=\infty$. This means that the singularity at $\sigma=0$ may be absorbed by a renormalization of the bare mass and bare charge. If one proceeds with Birkhoff's theorem and defines the mass-energy content inside the radius $r$ as the contributions of the combined matter and Electrostasic energy, this would allow 
us to evaluate the expression for $f(r)$ in terms of the EM mass-energy content $\mathcal{M}_{E M}(r)$ due to the Electrostatic energy density $E(r)^{2}$ associated with the electric field $E(r)$ :

$$
\begin{gathered}
\frac{e^{2} f(r)}{r^{2}} \equiv \frac{2 G \mathcal{M}_{E M}(r)}{r}=\frac{2}{8 \pi r} \quad \int_{0}^{r}[E(r)]^{2} 4 \pi r^{2} d r= \\
\frac{1}{4 \pi r} \int_{0}^{r}\left[\frac{q(r)}{r^{2}}\right]^{2} 4 \pi r^{2} d r=\frac{1}{4 \pi r} \int_{0}^{r}\left[\left(\frac{2 e}{\sqrt{\pi}} \gamma\left[\frac{3}{2} ; \frac{r^{2}}{4 \sigma^{2}}\right]\right)\left(\frac{1}{r^{2}}\right)\right]^{2} 4 \pi r^{2} d r .
\end{gathered}
$$

The integration can be carried out by a change of variables $(r / 2 \sigma)^{2}=x$. The relevant indefinite integral is given in terms of the error function er $f[x]$ and the incomplete upper Gammas $\Gamma[c, x]$, it is

$\int \gamma\left[\frac{3}{2}, x\right]^{2} x^{-3 / 2} d x=\frac{1}{2 \sqrt{x}} \sqrt{2 \pi x} \operatorname{erf}(\sqrt{2 x})-\frac{1}{2 \sqrt{x}} e^{-2 x}\left[-2 e^{x} \Gamma\left[\frac{3}{2}, x\right]+2 \sqrt{x}+e^{x} \sqrt{\pi}\right]^{2}$.

One can verify that the above integral expression at $x=0(r=0)$ is well behaved and is given by 0 , such that the lower limit of the integral in eq-(2.44) times the $1 / r$ pre-factor is finite at $r=0$. Therefore, there is no need to reabsorb infinities due to the $r=0$ contribution into a renormalization of the bare mass and bare charge, we refer to [21] for further details. We need to compare our result for the terms $e^{2} f(r) / r^{2}$ in eqs- $(2.44$, 2.45) based on Birkhoff's theorem with the results of (2.43) by [23]. A detailed graphical analysis of the behaviour of the $\sigma$-deformed Hawking temperature $T_{H}(\sigma)$ and the outer and inner deformed horizons can be found in [23]. Once more, one may evaluate the (Euclideanized) EM part of the action in the bulk domain bounded by the inner and outer deformed horizons $r_{ \pm}(\sigma)$ :

$$
S_{E}=\frac{1}{16 \pi G} \int_{r_{-}(\sigma)}^{r_{+}(\sigma)}[E(r, \sigma)]^{2}\left(4 \pi r^{2} d r\right) \int_{0}^{1 / k_{B} T(\sigma)} d t_{E} .
$$

The inner and outer $r_{ \pm}^{R N}(\sigma)$ deformed horizons are given in terms of solutions to a trascendental equation, and because they are not given any longer by analytical expressions, this entails the possibility of writing the definite integral $I^{R N}=I^{R N}\left[r_{+}(\sigma), r_{-}(\sigma)\right]$ corresponding to the deformed Reissner-Nordstrom case ( RN ) in terms of a power series expansion in the variable $\sigma$ of the form :

$$
S_{E}\left[A^{R N}(\sigma)\right]=\frac{A^{R N}(\sigma)}{4 G_{N}}+b^{R N}(\sigma) \log \frac{A^{R N}(\sigma)}{4 G_{N}}+\sum_{n=0}^{n=\infty} a_{n}^{R N}(\sigma)\left(\frac{A^{R N}(\sigma)}{4 G_{N}}\right)^{-n} .
$$

where $A(\sigma)=4 \pi\left(r_{+}^{R N}(\sigma)\right)^{2}$ and the $\sigma$-dependent coefficients $b(\sigma)$ and $a_{n}(\sigma)$ are defined implicitly by the integral $I^{R N}\left[r_{+}(\sigma), r_{-}(\sigma)\right]$. In the $\sigma \rightarrow 0$ limit one recovers the standard action-area-entropy relation in natural units $\hbar=c=k_{B}=1$ given by $S\left[A^{R N}(\sigma=0)\right]=$ $A^{R N}(\sigma=0) / 4 G_{N}=4 \pi\left(r_{+}^{R N}\right)^{2} / 4 G_{N}$ as shown by eq- $(2.30)$, since the inner and outer horizons and temperature will attain their undeformed values in the $\sigma=0$ limit.

The $\sigma$ deformed charged-rotating Kerr-Newman Black hole has not been constructed to our knowledge. One would proceed similarly to the $\sigma$-deformed Reissner-Nordstrom 
case. One would smear the rotating charged ring by an extended charged spinning object, like a spinning $p$-brane which behaves like a charged spinning fluid filling all of space and whose density profile is given by a Gaussian distribution. Another configuration besides the Gaussian might be modeled by Spinning Gravitational Skyrmions. To finalize this section, I have been informed by ${ }^{3}$ that the Spectroscopy of $\sigma$-deformed Black holes (quasi-normal modes expansion) can be studied by fluctuations of the location and shape of the horizon, in particular by fluctuations in the value of $\sigma$. For a recent treatment of Gravitational Entropy and Ricci-Finsler flows within the context of Grisha Perelman's work [30] we refer to [29], [28], [31].

\section{Why the use of the modulus $|r|$ is necessary to yield delta function terms}

The direct approach in solving Einstein's equation in the presence of a point mass delta function source requires replacing everywhere $r \rightarrow|r|$ in the radial gauge $R(|r|)=|r|+$ $2 G M \Theta(|r|)$. If one does not properly use $|r|$ ( instead of $r$ ) in the metric one will get an identically zero expression for the Einstein tensor as in the vacuum case. To illustrate how relevant it is to take the proper absolute values, we recall (in flat space) that the Laplacian in spherical coordinates of $1 /|r|$ is

$$
\begin{gathered}
\frac{1}{r^{2}}(d / d r)\left[r^{2}(d / d r)(1 /|r|)\right]=\frac{1}{r^{2}}(d / d r)\left[r^{2}\left(-1 /|r|^{2}\right) \operatorname{sign}(r)\right]= \\
-\frac{1}{r^{2}}(d / d r) \operatorname{sign}(r)=-\left(1 / r^{2}\right) \delta(r)
\end{gathered}
$$

since $r^{2}=|r|^{2}$, which is consistent with Poisson's law which states that the Laplacian of the Newtonian potential $-G M /|r|$ is $4 \pi G \rho$. This is true here if, and only if, $\rho=$ $\left(M / 4 \pi r^{2}\right) \delta(r)$ that is indeed the case in Newtonian gravity. To reiterate once more, the Laplacian in spherical coordinates of $(1 / r)$ is zero. For this reason, there is a fundamental difference in dealing with expressions involving absolute values $|r|$ like $1 /|r|$ from those which depend on $r$ like $1 / r$ [11].

Let us try to solve Einstein's equations for a point mass, firstly, by writing the compo-

nents of $T_{\mu \nu}$ associated with a point mass particle which is moving in its own gravitational background (neglecting the back reaction on the particle ) in terms of the appropriately defined covariantized delta function The worldline of the point mass source is parametrized by the four functions

$$
X^{0}=t(\tau), \quad X^{1}=r(\tau) ; \quad X^{2}=\theta(\tau) ; \quad X^{3}=\phi(\tau)
$$

The matter action is

$$
S_{\text {matter }}=-M_{o} \int d \tau=-M_{o} \int \sqrt{g_{\mu \nu}\left(d X^{\mu} / d \tau\right)\left(d X^{\nu} / d \tau\right)} d \tau=
$$

\footnotetext{
${ }^{3}$ J.F Gonzalez, private communication
} 


$$
-M_{o} \int \sqrt{g} d^{n} x \int \frac{\delta^{n}\left(x^{\mu}-X^{\mu}(\tau)\right)}{\sqrt{|g|}} \sqrt{g_{\mu \nu}\left(d x^{\mu} / d \tau\right)\left(d x^{\nu} / d \tau\right)} d \tau .
$$

From which we can deduce the expression for the stress energy tensor density

$$
\begin{gathered}
T^{\mu \nu}=-\frac{2}{\sqrt{g}} \frac{\delta S_{\text {matter }}}{\delta g_{\mu \nu}}= \\
M_{o} \int \frac{\left(d x^{\mu} / d \tau\right)\left(d x^{\nu} / d \tau\right)}{\sqrt{\left(d x^{\sigma} / d \tau\right)\left(d x_{\sigma} / d \tau\right)}} \frac{1}{\sqrt{|g|}} \delta(r-r(\tau)) \delta(\theta-\theta(\tau)) \delta(\phi-\phi(\tau)) \delta\left(t-x^{0}(\tau)\right) d \tau .
\end{gathered}
$$

The worldline of an inert point mass ( ignoring the back reaction of the gravitational field ) at fixed values of

$$
r=r_{o}=\text { constant } \neq 0 ; \quad \theta=\theta_{o}=\text { constant }, \quad \phi=\phi_{o}=\text { constant }
$$

is determined by the temporal function $x^{0}=t=x^{0}(\tau)$ such that

$$
\begin{gathered}
(d \tau)^{2}=g_{00}(d t)^{2} \Rightarrow \int \tau=\int \sqrt{g_{00}} d t \Rightarrow \frac{d t}{d \tau}=\frac{1}{\sqrt{g_{00}}} \\
\left(d x^{0} / d \tau\right)\left(d x^{0} / d \tau\right)=\frac{1}{g_{00}}=g^{00} .
\end{gathered}
$$

For this particular timelike worldline history (on-shell so $\left(d x^{\sigma} / d \tau\right)\left(d x_{\sigma} / d \tau\right)=1$ ) the only non-vanishing component of the stress energy tensor is

$$
\begin{gathered}
T_{00}=M_{o} \int \frac{\left(d x_{0} / d \tau\right)^{2}}{\sqrt{|g|}} \delta(r-r(\tau)) \delta(\theta-\theta(\tau)) \delta(\phi-\phi(\tau)) \frac{\delta\left(t-x^{0}(\tau)\right)}{\sqrt{\left(d x^{\sigma} / d \tau\right)\left(d x_{\sigma} / d \tau\right)}} d \tau= \\
T_{00}=M_{o} \int \frac{g_{00}\left(\left|\vec{r}-\vec{r}_{o}\right|\right)}{\sqrt{|g|}} \delta\left(r-r_{o}\right) \delta\left(\theta-\theta_{o}\right) \delta\left(\phi-\phi_{o}\right) \delta\left(t-x^{0}(\tau)\right) d \tau= \\
T_{00}=M_{o} \frac{g_{00}\left(\left|\vec{r}-\vec{r}_{o}\right|\right)}{\sqrt{|g|}} \delta\left(r-r_{o}\right) \delta\left(\theta-\theta_{o}\right) \delta\left(\phi-\phi_{o}\right) .
\end{gathered}
$$

As expected, we have found that the $T_{00}$ component is just related to the mass density $\rho$ in spherical ccordinates for a point mass source located at $\vec{r}_{o}=\left(x_{o}, y_{o}, z_{o}\right) \neq 0$. If the point mass source is located at the origin of the spherical coordinates system $\vec{r}_{o}=0$, the Jacobian in this case becomes $\sqrt{|g|}=R^{2}(d R / d r) \sin \phi$, and $g_{00}\left(\left|\vec{r}-\vec{r}_{o}\right|\right)=g_{00}(|r|)$. However, since the angles are degenerate at $r=0$ ( the angles are not well defined at the origin ) to cure this ambiguity one can perform the average over all solid angle directions ( from 0 to $4 \pi$ ) and which furnishes a crucial $(1 / 4 \pi$ ) factor that is deeply connected to the ubiquitous $2 M$ term, as follows

$$
\frac{1}{4 \pi} \int T_{00} \sin (\phi) d \phi d \theta=\frac{M_{o}}{4 \pi} \int \frac{g_{00}(r)}{R^{2}(d R / d r) \sin \phi} \delta(r) \delta\left(\theta-\theta_{o}\right) \delta\left(\phi-\phi_{o}\right) \sin (\phi) d \phi d \theta=
$$




$$
<T_{00}>_{\text {solid angle }}=g_{00}(r) \frac{M_{o}}{4 \pi R^{2}(d R / d r)} \delta(r) .
$$

Using the rules of differentiation outlined in eqs-(3.9, 3.10), the Einstein tensor, obtained by replacing $r \rightarrow|r|$ in the solutions (1.1), has non-vanishing diagonal elements involving a stress energy tensor with both pressure and density terms proportional to $\delta(r)$ [35]. However, despite this unexpected finding, we found that the integral of $8 \pi G$ times the trace of the stress energy tensor does satisfy the condition [35]

$$
\begin{gathered}
\int 8 \pi G_{N} \text { trace } T_{\mu \nu}=\int 8 \pi G_{N} g^{\mu \nu} T_{\mu \nu}=\int 8 \pi G_{N} g^{00}<T_{00}>= \\
-\int \mathcal{R}=\int \frac{2 G_{N} M_{o}}{R^{2}(r)(d R / d r)} \delta(r)\left(4 \pi R^{2}\right) d R d t=\int \frac{2 G_{N} M_{o}}{r^{2}} \delta(r)\left(4 \pi r^{2}\right) d r d t .
\end{gathered}
$$

in accordance to the results involving the integrals in the $\sigma \rightarrow 0$ limit of $(2.7 \mathrm{a}, 2.7 \mathrm{~b})$.

The scalar curvature associated with radial gauges involving the modulus $|r|$ (instead of $r$ ) generates the sought after $\delta(r)$ terms only in those expressions involving second derivatives of the metric. This is a consequence of

$$
\begin{gathered}
\frac{d g_{\mu \nu}(|r|)}{d r}=\frac{d g_{\mu \nu}(|r|)}{d|r|} \frac{d|r|}{d r}=\frac{d g_{\mu \nu}(|r|)}{d|r|} \operatorname{sign}(r) \Rightarrow\left(\frac{d g_{\mu \nu}(|r|)}{d r}\right)^{2}=\left(\frac{d g_{\mu \nu}(|r|)}{d|r|}\right)^{2} . \\
\frac{d^{2} g_{\mu \nu}(|r|)}{d r^{2}}=\frac{d^{2} g_{\mu \nu}(|r|)}{d|r|^{2}}+\frac{d g_{\mu \nu}(|r|)}{d|r|} \delta(r), \text { since sign }(r)^{2}=1, \quad \frac{d \operatorname{sign}(r)}{d r}=\delta(r) .
\end{gathered}
$$

Writing the metric components

$$
\begin{aligned}
g_{00}=1-\frac{2 G M}{|r|}=1-\frac{2 G M}{r} \frac{r}{|r|} & =1-\frac{2 G M}{r} f(r) ; \quad f(r) \equiv \frac{r}{|r|} . \\
g_{r r} & =-\frac{1}{g_{00}} .
\end{aligned}
$$

such that the derivatives

$$
f^{\prime}(r)=\frac{d f(r)}{d r}=\delta(r) ; \quad f^{\prime \prime}(r)=\frac{d^{2} f(r)}{d r^{2}}=\delta^{\prime}(r) .
$$

reveals that the nonvanishing $\mathcal{R}$ is given by :

$$
\begin{aligned}
\mathcal{R}= & -2 G M\left[\frac{f^{\prime \prime}(r)}{r}+2 \frac{f^{\prime}(r)}{r^{2}}\right]= \\
& -2 G M\left[\frac{\delta^{\prime}(r)}{r}+2 \frac{\delta(r)}{r^{2}}\right] .
\end{aligned}
$$

the signature chosen is $(+,-,-,-)$. 
Therefore, the Einstein-Hilbert action involving both density and pressure terms is exactly equal to an integral involving $2 G M \delta(r) / r^{2}$ :

$$
S=-\frac{1}{16 \pi G} \int \mathcal{R} 4 \pi r^{2} d r d t=\frac{1}{16 \pi G} \int 2 G M\left[\frac{\delta^{\prime}(r)}{r}+2 \frac{\delta(r)}{r^{2}}\right] 4 \pi r^{2} d r d t
$$

Integrating by parts yields

$$
\begin{gathered}
\frac{1}{16 \pi G} \int 8 \pi G M[2 \delta(r)-\delta(r)] d r d t=\frac{1}{16 \pi G} \int 8 \pi G\left(\frac{M \delta(r)}{4 \pi r^{2}}\right) 4 \pi r^{2} d r d t= \\
\frac{1}{16 \pi G} \int 8 \pi G \rho(r) 4 \pi r^{2} d r d t=\frac{1}{2} \int M d t \Rightarrow \rho(r) \equiv \frac{M \delta(r)}{4 \pi r^{2}}
\end{gathered}
$$

which is precisely the same result as the integral in eq-(2.10) . Notice that the authors [15] chose a very different function $f(r)=r^{\lambda}$ than the one chosen above $f(r)=r /|r|$, and in the limit $\lambda \rightarrow 0$, arrived at similar results for the distribution-valued scalar curvature. A different approach based on Colombeau's nonlinear distributional calculus was undertaken by $[14]$.

In showing why the integral of the trace of Einstein's equations corresponding to both density and pressure terms, given by eqs-(3.13, 3.14, 3.15), yields the same integral corresponding to the scalar curvature associated to a density and pressure terms given by eqs- $(2.9,2.10)$ is basically a similar exercise as integrating the Schroedinger equation in the presence of a delta function potential :

$$
\begin{gathered}
\int_{-\infty}^{+\infty}\left[-\frac{\hbar^{2}}{2 m} \frac{d^{2} \Psi}{d x^{2}}+\lambda \delta(x) \Psi(x)\right] d x=\int_{-\infty}^{+\infty} E \Psi d x \Rightarrow \\
\lambda \Psi(x=0)=E \int_{-\infty}^{+\infty} \Psi(x) d x .
\end{gathered}
$$

since the wave function and its derivative is required to obey the boundary conditions $\Psi(x= \pm \infty)=\Psi^{\prime}(x= \pm \infty)=0$ and normalized $\int|\Psi|^{2}=1$. The solution to (3.16) is $\Psi \sim e^{-k|x|}$ where $\lambda=2 E / k$. The matter density $\rho(r, \sigma)$ chosen by [22] is just the QM analog of the square $|\Psi|^{2}$ of a spherically symmetric Gaussian wave function $\Psi(r, \sigma(t))$ in 3 -dim. The $\sigma=0$ limit corresponds to a delta function localized source at $r=0$.

The black hole horizon can be displaced from $r_{h}=2 G M$ to a location arbitrarily close to $r=0$ by simply choosing the proper radial function $R=r+2 G M \Theta(r)$ with the correct behavior at $r=0$ given by $R(r=0)=0$. The novel metric which is not diffeomorphic to the metric in (3.11) due to the discontinuity at $r=0$, resulting from the definition of the Heaviside step function $\Theta(r)=1, r>0 ; \Theta(r=0)=0$ and $\Theta(r)=-1, r<0$, has for components

$$
g_{00}=1-\frac{2 G M}{|R|}=1-\frac{2 G M}{R} \frac{R}{|R|}=1-\frac{2 G M}{R} f(R) ; \quad f(R) \equiv \frac{R}{|R|}
$$




$$
g_{R R}=-\frac{1}{g_{00}}
$$

such that the nonvanishing $\mathcal{R}$ is given by :

$$
\begin{aligned}
\mathcal{R}= & -2 G M\left[\frac{f^{\prime \prime}(R)}{R}+2 \frac{f^{\prime}(R)}{R^{2}}\right]= \\
& -2 G M\left[\frac{\delta^{\prime}(R)}{R}+2 \frac{\delta(R)}{R^{2}}\right] .
\end{aligned}
$$

The Einstein-Hilbert action involving both density and pressure terms is exactly equal to an integral involving $2 G M \delta(R) / R^{2}$ :

$$
S=-\frac{1}{16 \pi G} \int \mathcal{R} 4 \pi R^{2} d R d t=\frac{1}{16 \pi G} \int 2 G M\left[\frac{\delta^{\prime}(R)}{R}+2 \frac{\delta(R)}{R^{2}}\right] 4 \pi R^{2} d R d t .
$$

Integrating by parts yields

$$
\begin{gathered}
\frac{1}{16 \pi G} \int 8 \pi G M[2 \delta(R)-\delta(R)] d R d t=\frac{1}{16 \pi G} \int 8 \pi G\left(\frac{M \delta(R)}{4 \pi R^{2}}\right) 4 \pi R^{2} d R d t= \\
\frac{1}{16 \pi G} \int 8 \pi G \rho(R) 4 \pi R^{2} d R d t=\frac{1}{2} \int M d t \Rightarrow \rho(R) \equiv \frac{M \delta(R)}{4 \pi R^{2}} .
\end{gathered}
$$

One learns also that the integrals are equal despite that the integrands and integration measures are not equal

$$
\frac{1}{16 \pi G} \int 8 \pi G\left(\frac{M \delta(R)}{4 \pi R^{2}}\right) 4 \pi R^{2} d R d t=\frac{1}{16 \pi G} \int 8 \pi G\left(\frac{M \delta(r)}{4 \pi r^{2}}\right) 4 \pi r^{2} d r d t
$$

Only for the Schwarzschild radial gauge $R^{3}=r^{3}+(2 G M)^{3}$, as a result of the condition $R^{2} d R=r^{2} d r$, and $\delta(R)=\delta(r) /(d R / d r)$ one can see that the integrands and measures in (3.21) are also the same. However, the Schwarzschild radial gauge is not correct since it leads to a contradiction due to the fact that it imposes a finite non-zero area condition for the point mass $A(r=0)=4 \pi R(r=0)^{2}=4 \pi(2 G M)^{2}$, while having a zero volume simultaneously. The point mass location is the center of spherical symmetry, and as such, cannot have a finite non-zero area. To cure this problem one must choose a radial gauge like $R=r+2 G M \Theta(r)$ to insure that $R(r=0)=0$ and such that the black hole horizon can be displaced from $r_{h}=2 G M$ to a location arbitrarily close to $r=0$. The metric is static everywhere, contrary to the Hilbert text-book solution that ceases to be static inside the horizon, and is discontinuous only at the location of the point mass singularity $r=0$ as expected.

Many still argue that the initial assumption of a point mass at $r=0$ is not physical. This is why we have modeled the delta function by a smeared Gaussian distribution and such that in the zero width limit one recover the same effects of the point mass source. A Gaussian wave function that begins as a delta function source localized at $r=0$, then 
it diffuses all over space but the center of the Gaussian remains fixed (static) at $r=0$ ( zero group velocity). The point mass at $r=0$ behaves as if it were delocalized all over space consistent with the wave-particle duality property in QM. To summarize : solutions invloving the modulus $|r|$ like $g_{\mu \nu}(|r|)$ are not diffeomorphic to those found by Hilbert $g_{\mu \nu}(r)$ and have a clear physical interpretation within the context of wave-particle duality in QM. A Gaussian mass distribution is inherently Quantum Mechanical when the mass density $\rho$ is the square of the wave function.

We end this section by analyzing the de Sitter vs Anti de Sitter behaviour of the metric in section 2.1 at small distances and the issue of negative temperature and entropy. It is true that the behaviour of $M(r, \sigma) / r$ for small values of $r$ behaves like $(1 / r)\left(r^{2} / 4 \sigma^{2}\right)^{3 / 2}$. Since one must not forget the square root present in the power $3 / 2$, there are plus or minus choices to consider. If one chooses the plus-sign branch of the square root one gets the de Sitter like behaviour of the metric $g_{t t} \sim 1-\lambda r^{2}$ at small scales found by [22]. But if one chooses the minus sign in front of the square root one will get the Anti de Sitter behaviour $g_{t t} \sim 1+\lambda r^{2}$. Both de Sitter and Anti de Sitter features have also been found by [44] in their work on the Renormalization Group improved Schwarzschild Black holes based on a running flow of the Newtonian coupling $G(r)$ where one, two and no horizons, were also obtained depending on the values of the mass $M_{o}$. The behaviour of $G(r)$ [44] at $r=0$ and $r \rightarrow \infty$ is similar to the one of $M(r, \sigma)[22] . G(r=0)=0$ and $G(r \rightarrow \infty) \rightarrow G_{N}$ whereas $M(r=0, \sigma)=0, M(r=\infty, \sigma)=M_{o}$ for a finite non-zero value of $\sigma$. In [43] we explored the case when both $G(r)$ and $M(r)$ vary with scale.

Finally, the location of the horizon at $r=2 G_{N} M$, or any other location for that matter, is a just a gauge artifact of the radial function one chooses to use. Choosing the step function radial gauge $R(r)=r+2 G M \Theta(r)$ or $R(r, \sigma)=r+r_{s}(\sigma) \Theta(r)$ to enforce $R(r=0)=0$, allows to shift the location of the horizon-to-be at $r=2 G_{N} M$ (or $r=r_{s}$ ) to $r=0$. Similar conclusions, a formation of a horizon at $r=0$ due to modifications of the black hole interior metric, have been found by [50] using Loop Quantum Gravity techniques (Loop Quantum Black Holes). Concluding, it is in this fashion, by this shifting of horizon locations, how we may avoid the problem of having negative temperatures and negative entropies and the like, because when one tries to go lower than $r=0$, one has entered into the white-hole solution region $r<0$ of repulsive gravity. Nevertheless this does not mean that non-extensive Tsallis statistics or exotic q-statics in Fractal spacetimes are not relevant in the future as viable solutions to the problem of negative entropy and temperature [41]. The interplay between Non-extensive statistics, Chaos and fractal strings was studied in [55].

\section{Stringy Black Holes, Finsler Geometry and Maxi- mal Acceleration in Phase Spaces}

Another possibility to study the field of a point mass is to formulate the problem in phase space, in particular within the framework of the Finsler geometry associated with 
the (co) tangent bundle of spacetime. A point mass may have a zero area from the space-time perspective but a non-zero area from the phase space point of view due to the incorporation of the momentum degrees of freedom into the picture; i.e. in the static case $p^{\mu}=(E=M, 0,0,0)$ there is a non-vanishing phase space area element ( setting aside the nature of curved phase space for the moment ) $\mathcal{A}=E t=M t$. A compactification of the temporal direction $t$ along a circle $S^{1}$ gives an Euclidean time coordinate interval of $2 \pi t_{E}$ which is defined in terms of the Hawking temperature $T_{H}$ and Boltzman constant $k_{B}$ as $2 \pi t_{E}=\left(1 / k_{B} T_{H}\right)=8 \pi G_{N} M$. From which we infer that $t_{E}=4 G_{N} M$. Therefore the area element in phase space, $\mathcal{A}$, after equating $G_{N}=L_{\text {Planck }}^{2}$, (in natural units $\hbar=c=1$ ) is

$$
\mathcal{A}=E t_{E}=M \times 4 G_{N} M=4 G_{N} M^{2}=\frac{4 \pi\left(2 G_{N} M\right)^{2}}{4 \pi L_{P}^{2}}=\frac{\text { Horizon Area }}{4 \text { Planck Disc }} .
$$

where the area of the Planck disc is $\pi\left(L_{P}\right)^{2}$. Therefore, the phase space area element $E t_{E}$, in units of $\hbar$, is the same as the Black Hole Entropy ( one quarter of the area of the spherical horizon at $r=2 G_{N} M$ ) in units of the area of a Planck disc. This fact may have some relation to the Holographic principle and warrants investigation.

In [43] a natural cut-off of the form $R(r=0)=2 G_{N} M$ was interpreted from the standard Noncommutative spacetime coordinates algebra $\left[x^{\mu}, x^{\nu}\right]=i \Sigma^{\mu \nu},\left[p^{\mu}, p^{\nu}\right]=$ $0,\left[x^{\mu}, p^{\nu}\right]=i \hbar \eta^{\mu \nu}$ where $\Sigma^{\mu \nu}$ are $c$-numbers of $(\text { Planck length })^{2}$ units. A change of coordinates in phase space $x^{\prime \mu}=x^{\mu}+\frac{1}{2} \Sigma^{\mu \nu} p_{\nu}$ leads to commuting coordinates $x^{\prime \mu}$ and allows to define $r^{\prime}(r)=\sqrt{\left(x^{i}+\frac{1}{2} \Sigma^{i \rho} p_{\rho}\right)\left(x_{i}+\frac{1}{2} \Sigma_{i \tau} p^{\tau}\right)}$. One can select $\Sigma^{\mu \nu}$ such that $r^{\prime}\left(x^{i}=0\right)=r^{\prime}(r=0)=2 G_{N} M_{o}$, upon using $p_{\mu} p^{\mu}=M_{o}^{2}$ in the static case $p^{\mu}=\left(M_{o}, 0,0,0\right)$ [43] which is precisely the cut-off corresponding to the AbramsSchwarzschild radial gauge. Noncommutative Finsler gravity ( Lagrange-Finsler manifolds ) associated with the spacetime tangent bundle and the Hamilton-Cartan geometry of Noncommutative phase spaces, is the arena where properly one can study the Noncommutative Gravity of the spacetime tangent ( co-tangent ) bundle [27].

Since coordinates and velocities (momenta in phase spaces ) are treated on equal footing, Lagrange-Finsler ( Hamilton-Cartan ) geometry is the backdrop where one may achieve a Geometrization of matter and have a space-time-matter unity. The natural group acting in phase spaces is $U(1,3)=S U(1,3) \times U(1)$ to account for acceleration and boosts transformations. The maximal proper force (acceleration) postulated by Max Born many years ago that a fundamental particle may experience is $m_{\text {Planck }} c^{2} / L_{\text {Planck }}$. The group $S U(1,3)$ is not the same as the conformal group $S U(2,2)$. Finsler spaces have torsion which is the hallmark of spin. An entirely different approach to treat point mass delta function source can be found in [42].

A Planck scale cut-off can be derived in terms of noncommutative Moyal star products $f(x) * g(x)$ simply by replacing $r \rightarrow r_{*}=\sqrt{r * r}=\sqrt{r^{2}+\sum^{i j} x_{i} x_{j} / r^{2}+\ldots}$ so $r_{*}\left(x^{i}=\right.$ $0) \neq 0$, and receives Planck scale corrections. A point is fuzzy and delocalized, henceforth it has a non-zero fuzzy area and fuzzy volume. An open problem is to verify whether or not Schwarzschild deformed metrics of the form 


$$
g_{t t}\left(r_{*}\right)=1-\frac{2 G M}{r_{*}}, \quad g_{r r}=-g_{t t}^{-1}, \quad r_{*}=\sqrt{r * r}=\sqrt{r^{2}+\Sigma^{i j} x_{i} x_{j} / r^{2}+\ldots} .
$$

with the angular part $r_{*} * r_{*}(d \Omega)^{2}$, solve the Noncommutative Gravity field equations to all orders in the noncommutative parameter $\Sigma^{\mu \nu}$. A p-Adic norm naturally attains the feature of delocalization since a p-Adic disc has no center. Every point $i s$ the center. Notice the difference between the latter Planck scale cut-off in ordinary spacetime with the former mass (momentum) dependent cut-off $2 G_{N} M_{o}$ in the spacetime tangent ( cotangent ) bundle. Yang's Noncommutative algebra in phase space, has both an ultraviolet and infrared cut-off related to the minimal ( Planck) and maximal (Hubble) scale. A Moyal-Fedosov-Kontsevich star products deformations of $p$-branes were constructed in [33] based on Yang's algebra.

We will show in this section how the horizon of the standard black hole solution at $r=2 G_{N} M$ ( when the Hilbert textbook choice is taken $R(r)=r$ ) can be displaced to the location of the point mass $r=0$, when the radial function is chosen to have a cutoff $R(r=0)=2 G_{N} M$, if, and only if, one embeds the problem in phase space ( or the spacetime tangent bundle) that is the proper arena to incorporate the role of the physical point mass $M$ at $r=0$. Relativity in phase space is the arena where one may unify space, time and matter due to the equivalence between mass and energy. In the Kruskal-Fronsdal-Szekeres coordinates $u, v$ description, to describe what happens when one crosses the horizon $r=2 G_{N} M$ of topology $R \times S^{2}$ and whose spatial slice is a sphere of radius $r=2 G M$, one has a null hyper-surface at $r=2 G_{N} M$ due to the tipping of the lightcone as one approaches the horizon. When the radial function obeys a different boundary condition $R(r=0)=2 G_{N} M$ than the Hilbert textbook one, one may displace the null horizon from $r=2 G_{N} M$ to a null horizon in $r=0$ but such horizon lives in the spacetime tangent bundle (phase space) to account for the presence of a point-mass $M$ at $r=0$. Furthermore, to corroborate our proposal, we will show the relationship between our description of the field of a point mass, within the framework of phase spaces (Hamilton-Cartan Geometry ) or in the spacetime tangent bundle (Finsler geometry), and the Bars-Witten stringy black hole in $1+1$-dim that has a null horizon at $r=0$. The stringy black hole singularity occurs in the complex realm when $r$ is extended to the field of complex numbers $r=0+i\left(2 G_{N} M\right)(\pi / 2)$. Whereas the horizon ( the null surface ) actually lives at $r=0$.

The physical motivation of embedding the problem in a larger space (phase space ) was already evisioned by Max Born [57] who was the first to propose a Reciprocal ( or Dual ) Relativity Principle in Phase Spaces, where in addition to a limiting speed given by the speed of light, there is a limiting proper force (acceleration). Since speed is the rate of change of position, and force is the rate of change of momentum, then the reciprocal principle of Relativity in Phase Spaces requires a limiting speed given by the speed of light and a maximal force experienced by a fundamental particle that can be conjectured to be $F=m_{\text {Planck }} c^{2} / L_{\text {Planck }}=M($ Universe $) c^{2} / R_{\text {Hubble }}$ and which leads to the WeylDirac-Eddington large numbers coincidence in Cosmology [59]. A maximal acceleration $c^{2} / L_{\text {Planck }}$ is also consistent with the Finsler geometry of the spacetime tangent bundle [58] and the stringy minimal Planck length uncertainty relations [60] 


$$
\Delta X \geq \frac{\hbar}{\Delta P}+L_{\text {Planck }}^{2} \Delta P .
$$

The most general $p$-brane uncertainty relations based on a unified treatment of $p$-branes, for all values of $p$, in Clifford spaces was derived in [61].

The physical interpretation of the phase space null horizon at $r=0$, null from the perspective of the full fledged phase space metric $g_{\mu \nu}(x, p)$, or Finsler metric $g_{\mu \nu}(x, v)$ is that it is the "attractor" region where a test particle (of mass $m$ ) approaches asymptotically as it moves in the gravitational background produced by the point mass $M$ located at $r=0$ (when $m<<<M$ ). As the test particle approaches the horizon at $r=0$, its speed and acceleration approach asymptotically the speed of light $c$ and the maximal acceleration $c^{2} / L_{P}$. This is very reminiscent of what occurs when one uniformly accelerates a massive test particle in flat Minkowski spacetime, the trajectory is a hyperbola which asymptotically approaches the light cone passing through the Minkowski spacetime origin $r=0$. The speed tends asymptotically to the speed of light.

We will explain now why the Bars-Witten stringy $1+1$-dim black-hole solution can be embedded into the conformally re-scaled 4-dim metrics of the form in eq-(1.1) for a unique choice of the radial function given by the tortoise radial coordinate [45]

$$
R+2 G_{N} M \ln \left(\frac{R-2 G_{N} M}{2 G_{N} M}\right)=2 G_{N} M \ln \left[\sinh \frac{r}{2 G_{N} M}\right] .
$$

such that

$$
R(r=0)=2 G_{N} M ; \quad R(r \rightarrow \infty) \rightarrow R \sim r .
$$

The radial function $R(r=0)=2 G_{N} M$ has also a lower ( ultraviolet cutoff ) bound given by $2 G_{N} M$. An interesting analysis of how a string ( an extended object ) can probe space-time points was presented by Aspinwall [53]. This requires altering our classical conceptions of Topology and Geometry at very small scales.

The Bars-Witten black-hole $1+1$-dim metric ( setting $2 G M=1$ ) is :

$$
d s^{2}=(d r)^{2}-\tanh ^{2}(r)(d t)^{2}=-\frac{d u d v}{1-u v} .
$$

with

$$
u=\frac{1}{2} \exp \left[r+t+\log \left(1-e^{-2 r}\right)\right] ; \quad v=-\frac{1}{2} \exp \left[r-t+\log \left(1-e^{-2 r}\right)\right] .
$$

the Euclidean analytical continuation of the metric in eq-(4.5) is obtained by setting $\theta=i t$, such that the metric is $d s^{2}=d r^{2}+\tanh ^{2} r d \theta^{2}$ and its Euclidean geometry has the shape of a semi-infinite cigar that asymptotically approaches $R^{1} \times S^{1}$ for $r \rightarrow \infty$. We should notice that the Lorentzian metric of eq-(4.5) has a singularity at a complex value $r=0+i \pi / 2($ setting $2 G M=1)$ since $\tanh ^{2}(i \pi / 2)=-\tan ^{2}(\pi / 2)=-\infty$ which is consistent with the singularities at the location where $u v=-\frac{1}{4} e^{2 r}\left(1-e^{-2 r}\right)^{2}=1$, when $r=0+i \pi / 2$, and a horizon at $r=0$, since $u v=0$ when $r=0$. 
However this is not the end of the story. The Bars-Witten black hole in $1+1$-dim is obtained from a gauged $S l(2, R) / U(1)$ WZNW model with central charge $c=2+6 / k$ and is a consistent bosonic string background solution in a $1+1$ target background given by the two-dim coset $S l(2, R) / U(1)$. Namely, the CFT corresponding to the gauged $S l(2, R) / U(1)$ WZNW model with central charge $c=2+6 / k i s$ a solution of equations derived from the vanishing beta functions required by conformal invariance of the non-linear sigma model. For example, the relevant massless bosonic closed-string fields in a $D=26$ dim target background ( a different CFT ) are the antisymmetric tensor $B_{\mu \nu}\left(X^{\rho}\left(\sigma^{a}\right)\right)$; the dilaton $\Phi\left(X^{\rho}\left(\sigma^{a}\right)\right)$ and the gravitational field $\left.g_{\mu \nu}\left(X^{\rho}\left(\sigma^{a}\right)\right)\right)$; where $\sigma^{a}=\sigma^{1}, \sigma^{2}$ are the world-sheet variables. The conditions for the vanishing of the one loop beta functions, required by Weyl invariance of the non-linear sigma model, to leading order in the string tension $\alpha^{\prime}$ turn out to be [52]

$$
\begin{gathered}
\mathcal{R}_{\mu \nu}+\frac{1}{4} H_{\mu}^{\lambda \rho} H_{\nu \lambda \rho}-2 D_{\mu} D_{\nu} \Phi=0 . \\
D_{\lambda} H_{\mu \nu}^{\lambda}-2\left(D_{\lambda} \Phi\right) H_{\mu \nu}^{\lambda}=0 . \\
4\left(D_{\mu} \Phi\right)^{2}-4 D_{\mu} D^{\mu} \Phi+\mathcal{R}+\frac{1}{12} H_{\mu \nu \rho} H^{\mu \nu \rho}=0 .
\end{gathered}
$$

where

$$
H_{\mu \nu \rho}=\partial_{\mu} B_{\nu \rho}+\partial_{\rho} B_{\mu \nu}+\partial_{\nu} B_{\rho \mu} .
$$

is the third rank antisymmetric tensor field strength that is invariant under the transformations $\delta B_{\mu \nu}=\partial_{\mu} \Lambda_{\nu}-\partial_{\nu} \Lambda_{\mu}$.

Nevertheless, we will show how the Bars-Witten $1+1$-dim black hole metric can be embedded into the $3+1$-dim solutions of the appendix [45], up to a conformal factor $e^{\Upsilon}$ , since the latter metrics were Ricci flat by construction. The embedding of the $1+1$-dim metric (4.5) into the conformally re-scaled 3+1-dim solutions of the appendix are obtained by introducing the mass parameter $2 M$ ( in units of $G=c=1$ ) in the appropriate places in order to have consistent units, and by writing :

$$
e^{\Upsilon(r)}\left(1-\frac{2 M}{R(r)}\right)=\tanh ^{2}\left(\frac{r}{2 M}\right) ; \quad e^{\Upsilon(r)} \frac{(d R / d r)^{2}}{1-2 M / R(r)}=1 .
$$

leading to the solutions for $\Upsilon(r)$ and $R(r)$ respectively

$$
e^{\Upsilon}=\frac{1}{1-2 M / R(r)} \tanh ^{2}\left(\frac{r}{2 M}\right) .
$$

where

$$
\begin{gathered}
\int \frac{d R}{1-2 M / R}=R+2 M \ln \left(\frac{R-2 M}{2 M}\right)=\int \frac{d r}{\tanh r / 2 M}= \\
2 M \ln \left[\sinh \frac{r}{2 M}\right] .
\end{gathered}
$$

this last equation (4.9b) yields the functional form $R(r)$ ( tortoise radial variable ) in implicit form for the radial function $R(r)$. From eq- $(4.9 \mathrm{~b})$ one can infer that ( in units $c=G=1$ ) 


$$
R(r=0)=2 M ; \quad R(r \rightarrow \infty) \rightarrow R \sim r .
$$

The radial function $R$ has a lower ( ultraviolet cutoff ) bound given by $2 M$. The fact that the "point" $r=0$ can have a non-zero proper area but zero volume seems to indicate a "stringy" nature underlying the very notion of a point-mass itself. The string worldsheet has area but no volume. Aspinwall [53] has studied how a string ( an extended object ) can probe space-time "points".

Notice that if we allow for complex values of $r$, like $r=0+i 2 M(\pi / 2)$, that furnish singularities in the metric (4.5), one must include a constant of integration $R_{0}=2 M(1+$ $i \pi / 2)$ to the solution in eq- $(4.9 \mathrm{~b})$

$$
R-2 M(1+i \pi / 2)+2 M \ln \left(\frac{R-2 M}{2 M}\right)=2 M \ln \left[\sinh \frac{r}{2 M}\right] .
$$

such that when one plugs in the value $r=0+i 2 M(\pi / 2)$ in the right hand side of eq(4.11), it coincides with the left hand side of (4.11) when the value of the radial function $R(r=0+i 2 M \pi / 2)=2 M(1+i \pi / 2)$, after an analytical continuation into the complex plane is performed. This is just a consequence of the relation $\ln [\sinh (i \pi / 2)]=$ $\ln [i \sin (\pi / 2)]=\ln i=i \pi / 2$.

This complex analytical continuation into regions where $r, R$ are complex-valued roughly speaking amounts to looking into the "interior" of the point-mass. Having complex coordinates to probe into the "interior" of a point-mass is not so farfetched. This suggests that Quantum spacetime might be intrinsically fractal, meaning that the Hausdorff topological dimension of an object ( let us say of a point ) does not coincide with the fractal dimension. For a throrough and profound treatment of complex dimensions, fractal strings and the zeros of Riemman zeta function see [54]. The interplay among non-extensive statistics, chaos, complex dimensions, logarithmic periodicity in the renormalization group and fractal strings see [55].

The conformal factor is

$$
e^{\Upsilon}=\frac{1}{1-2 M / R(r)} \tanh ^{2}\left(\frac{r}{2 M}\right)
$$

where $R(r)$ is given implicitly by (4.9b). Notice that from the conditions in (4.10) the conformal factor $e^{\Upsilon}$ becomes unity at $r=\infty$ as it should if one wishes to have asymptotic flatness. When $r=0$ the conformal factor (4.12) is $\frac{0}{0}$ undefined. A careful study reveals that the conformal factor $e^{\Upsilon}$ at $r=0$ is zero so that $e^{\Upsilon(r=0)} R^{2}(r=0)=0$ and the conformally re-scaled proper area at $r=0$ is zero. Therefore, at $r=0$ the conformally rescaled interval $d s^{2}$ is zero consistent with the fact that the $1+1$-dim metric exhibits a null horizon at $r=0$. Concluding, in this fashion, we have shown how one can embed the 1+1-dim Bars-Witten stringy black hole solution into the conformally re-scaled 3+1-dim solutions of section of the appendix and are given by :

$$
d s^{2}=-\tanh ^{2}\left(\frac{r}{2 M}\right)(d t)^{2}+(d r)^{2}+e^{\Upsilon(r)} R^{2}(r) d \Omega^{2} .
$$


Notice that the conformally re-scaled metric (4.13) is not Ricci flat; it has singularities at complex values $r=0+i 2 M \pi / 2 \Rightarrow e^{\Upsilon}=\infty ; R=2 M(1+i \pi / 2)$ upon using eq-(4.11). There is a difference between the metric (4.13) with the Ricci flat metric ( outside the singularity at the point mass source ) given in the Fronsdal-Kruskal-Szekeres coordinates by

$$
\begin{gathered}
d s^{2}=-e^{W(u, v)} \frac{d u d v}{1-u v}+\left(R_{*}(u, v)\right)^{2}\left[\sin ^{2} \phi(d \theta)^{2}+(d \phi)^{2}\right]= \\
-e^{W(u, v)} \frac{d u d v}{1-u v}+\left(R_{*}(u, v)\right)^{2} d \Omega^{2}
\end{gathered}
$$

where $W(u, v)$ and $R_{*}(u, v)$ are now two complicated functions of the two variables $u, v$ ( since when one crosses the horizon the metric is no longer static). Whereas in eq-(4.13) one truly has a static metric everywhere and two functions of one variable $\Upsilon(r), R(r)$ instead.

Having discussed the stringy black hole, we turn to Finsler geometry. The geometric proper displacement in the spacetime tangent bundle involving coordinates and velocities typical of Finsler metrics is

$$
\begin{gathered}
(d \sigma)^{2}=(d s)^{2}+L_{P}^{2}\left(d v^{\mu}\right)^{2}=(d s)^{2}\left[1+L_{P}^{2}\left(\frac{d v^{\mu}}{d s}\right)^{2}\right]=\left(1-\frac{a^{2}(s)}{a_{o}^{2}}\right)(d s)^{2} . \\
-a^{2}(s) \equiv\left(\frac{d v^{\mu}}{d s}\right)^{2}<0 .
\end{gathered}
$$

the acceleration is spacelike when the velocity is timelike, this accounts for the minus sign in the last term of (4.15).

We are naturally assuming that the test particle does not follow a geodesic in the base spacetime manifold, otherwise $a=0$. For example, when the test particle remains static, the acceleration is the force per unit mass required to maintain the test particle at a fixed position ( since the metric is static ) and prevent it from falling into the point mass $M$ source at $r=0$. The closer it gets to $r=0$, the greater the force is required to hold it in that place. The force ( acceleration ) has an upper limit in our case due to Born's relativity principle in phase space. If one ignores the back-reaction of the gravitational field on the point mass $M$ at $r=0$, the world line of the very own point mass $M$, as it is inmersed in its own gravitational field background, is a timelike geodesic at $r=0$ such it does not experience an acceleration. So the world line interval corresponding to the point mass location $(d \sigma)^{2}$ coincides with $(d s)^{2}$ in this case.

The conformal factor in front of the standard spacetime interval $(d s)^{2}$ vanishes when the acceleration of the test particle moving in such background is $a^{2}=a_{o}^{2}$, where $a_{o}$ is the maximal acceleration $c^{2} / L_{\text {Planck }}$. When $(d s)^{2}$ is given by the Schwarzschild solutions of eq-(2.1) and when the radial functions $R(r)$ is subjected to the cutoff $R(r=0)=$ $2 G_{N} M$ we have a divergence of $(d s)^{2}(r=0)=\infty$, due to $g_{r r}(r=0)=\infty$, while the conformal factor is zero, since a test particle attains the limiting upper value of the proper acceleration when it asymptotically reaches $r=0$. Its speed also asymptotically tends to 
the speed of light as it approaches $r=0$. One then ends up with an interval $(d \sigma)^{2}$ of the form $0 \times \infty$ which nevertheless tends to zero.

The most salient feature of (4.15) is that at $r=0$ we end up with

$$
\left(1-\frac{a^{2}(s)}{a_{o}^{2}}\right)(d s)^{2}=0 \times \infty \rightarrow 0 .
$$

implying that the conformally rescaled Schwarzschild metrics (1.1) yield a null interval, a null surface $(d \sigma)^{2}=0$, in the spacetime tangent bundle at the precise location of the point mass source $r=0$. In the stringy black hole case the location $r=0$ is also a null surface and coincides with the Bars-Witten stringy black hole horizon. Hence, the horizon that a test particle ( of mass $m<<M$ ) experiences as it approaches $r=0$ asymptotically, is a null surface that lives in the spacetime tangent bundle corresponding to the coordinates $x^{\mu}(s), v^{\mu}(s)$ associated with the world-line of the test particle. This is

because the conformally rescaled area $\left(1-\frac{a^{2}(s)}{a_{o}^{2}}\right) 4 \pi R(r)^{2}$ that the test particle sees, as it approaches $r=0$, tends to zero due to the vanishing of the conformal factor when the maximal acceleration is attained, despite the fact that $4 \pi(R(r=0))^{2}=4 \pi\left(2 G_{N} M\right)^{2} \neq 0$. This is a peculiar feature of Finsler geometry when a metric is velocity ( momentum ) dependent in addition to position dependent.

To see why phase space metrics can behave like stringy black hole metrics, let us look for the analog of a static spherically symmetric metric in phase space ( or in the spacetime tangent bundle), which is for example, $g_{t t}\left(r, p_{r}\right) ; g_{r r}\left(r, p_{r}\right)$ where $p_{r}$ is the radial conjugate momentum to the radial variable $r$. This dependence on the conjugate pair of variables $\left(r, p_{r}\right)$ resembles the Bars-Witten stringy-black hole metric $(d s)^{2}=g_{u v}(u, v) d u d v$ depending on the pair of variables $u, v$. Thus, the maximal proper acceleration $a_{o}=$ $c^{2} / L_{\text {Planck }}$ acts as regulator in spacetime [58] in the same vein that there is a maximum value of tidal forces ( acceleration ) in string theory [60] due to the minimal length string uncertainty relations. This maximal acceleration regulator is consistent with the introduction of an ultra-violet cutoff $R(r=0)=2 G_{N} M$.

\section{Appendix A: Schwarzschild-like solutions in any di- mension $D>3$}

In this Appendix we follow closely our prior calculations [45]. Let us start with the line element

$$
d s^{2}=-e^{\mu(r)}\left(d t_{1}\right)^{2}+e^{\nu(r)}(d r)^{2}+R^{2}(r) \tilde{g}_{i j} d \xi^{i} d \xi^{j} .
$$

Here, the metric $\tilde{g}_{i j}$ corresponds to a homogeneous space and $i, j=3,4, \ldots, D-2$ and the temporal and radial indices are denoted by 1,2 respectively. In our text we denoted the temporal index by 0 . The only non-vanishing Christoffel symbols are

$$
\begin{array}{lll}
\Gamma_{21}^{1}=\frac{1}{2} \mu^{\prime}, & \Gamma_{22}^{2}=\frac{1}{2} \nu^{\prime}, & \Gamma_{11}^{2}=\frac{1}{2} \mu^{\prime} e^{\mu-\nu}, \\
\Gamma_{i j}^{2}=-e^{-\nu} R R^{\prime} \tilde{g}_{i j}, & \Gamma_{2 j}^{i}=\frac{R^{\prime}}{R} \delta_{j}^{i}, & \Gamma_{j k}^{i}=\tilde{\Gamma}_{j k}^{i},
\end{array}
$$


and the only nonvanishing Riemann tensor are

$$
\begin{array}{ll}
\mathcal{R}_{212}^{1}=-\frac{1}{2} \mu^{\prime \prime}-\frac{1}{4} \mu^{\prime 2}+\frac{1}{4} \nu^{\prime} \mu^{\prime}, & \mathcal{R}_{i 1 j}^{1}=-\frac{1}{2} \mu^{\prime} e^{-\nu} R R^{\prime} \tilde{g}_{i j}, \\
\mathcal{R}_{121}^{2}=e^{\mu-\nu}\left(\frac{1}{2} \mu^{\prime \prime}+\frac{1}{4} \mu^{\prime 2}-\frac{1}{4} \nu^{\prime} \mu^{\prime}\right), & \mathcal{R}_{i 2 j}^{2}=e^{-\nu}\left(\frac{1}{2} \nu^{\prime} R R^{\prime}-R R^{\prime \prime}\right) \tilde{g}_{i j}, \\
\mathcal{R}_{j k l}^{i}=\tilde{R}_{j k l}^{i}-R^{\prime 2} e^{-\nu}\left(\delta_{k}^{i} \tilde{g}_{j l}-\delta_{l}^{i} \tilde{g}_{j k}\right) . &
\end{array}
$$

The field equations are

$$
\begin{gathered}
\mathcal{R}_{11}=e^{\mu-\nu}\left(\frac{1}{2} \mu^{\prime \prime}+\frac{1}{4} \mu^{\prime 2}-\frac{1}{4} \mu^{\prime} \nu^{\prime}+\frac{(D-2)}{2} \mu^{\prime} \frac{R^{\prime}}{R}\right)=0, \\
\mathcal{R}_{22}=-\frac{1}{2} \mu^{\prime \prime}-\frac{1}{4} \mu^{\prime 2}+\frac{1}{4} \mu^{\prime} \nu^{\prime}+(D-2)\left(\frac{1}{2} \nu^{\prime} \frac{R^{\prime}}{R}-\frac{R^{\prime \prime}}{R}\right)=0,
\end{gathered}
$$

and

$$
\mathcal{R}_{i j}=\frac{e^{-\nu}}{R^{2}}\left(\frac{1}{2}\left(\nu^{\prime}-\mu^{\prime}\right) R R^{\prime}-R R^{\prime \prime}-(D-3) R^{\prime 2}\right) \tilde{g}_{i j}+\frac{k}{R^{2}}(D-3) \tilde{g}_{i j}=0,
$$

where $k= \pm 1$, depending if $\tilde{g}_{i j}$ refers to positive or negative curvature. From the combination $e^{-\mu+\nu} \mathcal{R}_{11}+\mathcal{R}_{22}=0$ we get

$$
\mu^{\prime}+\nu^{\prime}=\frac{2 R^{\prime \prime}}{R^{\prime}}
$$

The solution of this equation is

$$
\mu+\nu=\ln R^{\prime 2}+a
$$

where $a$ is a constant.

Substituting (A.7) into the equation (A.6) we find

$$
e^{-\nu}\left(\nu^{\prime} R R^{\prime}-2 R R^{\prime \prime}-(D-3) R^{\prime 2}\right)=-k(D-3)
$$

or

$$
\gamma^{\prime} R R^{\prime}+2 \gamma R R^{\prime \prime}+(D-3) \gamma R^{\prime 2}=k(D-3)
$$

where

$$
\gamma=e^{-\nu}
$$

The solution of (A.10) for an ordinary $D$-dim spacetime ( one temporal dimension ) corresponding to a $D-2$-dim sphere for the homogeneous space can be written as

$$
\gamma=\left(1-\frac{16 \pi G_{D} M}{(D-2) \Omega_{D-2} R^{D-3}}\right)\left(\frac{d R}{d r}\right)^{-2} \Rightarrow
$$




$$
g_{r r}=e^{\nu}=\left(1-\frac{16 \pi G_{D} M}{(D-2) \Omega_{D-2} R^{D-3}}\right)^{-1}\left(\frac{d R}{d r}\right)^{2} .
$$

where $\Omega_{D-2}$ is the appropriate solid angle in $D-2$-dim and $G_{D}$ is the $D$-dim gravitational constant whose units are $(\text { length })^{D-2}$. Thus $G_{D} M$ has units of $(\text { length })^{D-3}$ as it should. When $D=4$ as a result that the 2-dim solid angle is $\Omega_{2}=4 \pi$ one recovers from eq-(A.12) the 4-dim Schwarzchild solution. The solution in eq-(A.12) is consistent with Gauss law and Poisson's equation in $D-1$ spatial dimensions obtained in the Newtonian limit.

For the most general case of the $D-2$-dim homogeneous space we should write

$$
-\nu=\ln \left(k-\frac{\beta_{D} G_{D} M}{R^{D-3}}\right)-2 \ln R^{\prime}
$$

where $\beta_{D}$ is a constant. Thus, according to (A.8) we get

$$
\mu=\ln \left(k-\frac{\beta_{D} G_{D} M}{R^{D-3}}\right)+\text { constant } .
$$

we can set the constant to zero, and this means the line element (A.1) can be written as

$$
d s^{2}=-\left(k-\frac{\beta_{D} G_{D} M}{R^{D-3}}\right)\left(d t_{1}\right)^{2}+\frac{(d R / d r)^{2}}{\left(k-\frac{\beta_{D} G_{D} M}{R^{D-3}}\right)}(d r)^{2}+R^{2}(r) \tilde{g}_{i j} d \xi^{i} d \xi^{j} .
$$

One can verify, taking for instance (A.5), that the equations (A.4)-(A.6) do not determine the form $R(r)$. It is also interesting to observe that the only effect of the homogeneous metric $\tilde{g}_{i j}$ is reflected in the $k= \pm 1$ parameter, associated with a positive ( negative ) constant scalar curvature of the homogeneous $D-2$-dim space. $k=0$ corresponds to a spatially flat $D-2$-dim section.

The stress energy tensor for a point mass source is given explicitly by the zero-width limit of the Gaussian in the right hand side of eqs-(2.1,2.2), as shown explicitly in eqs(2.4), (2.9) and (2.10). Let us now verify that the line element (2.5) is a solution of Einstein's equations (2.1) in the presence of a mass distribution density $\rho(r)$. The temporal components of (2.1) yield

$$
\mathcal{R}_{00}-\frac{1}{2} g_{00} \mathcal{R}=e^{\mu-\nu}\left[\frac{\nu^{\prime}}{r}-\frac{1}{r^{2}}\right]+\frac{e^{\mu}}{r^{2}} .
$$

Defining the new solutions corresponding to the mass distribution $M(r, \sigma)$ by

$$
e^{\mu}=1-\frac{2 G_{N} M(r, \sigma)}{r} ; \quad e^{\nu}=e^{-\mu} ; \quad \mu=-\nu=\log \left(1-\frac{2 G_{N} M(r, \sigma)}{r}\right) .
$$

inserting eqs-(A.16, A.17) into the temporal components of eq-(2.1), and after factoring out the metric component $g_{00}(r)=e^{\mu}$, it becomes

$$
\frac{2 G_{N}}{r^{2}}\left(\frac{d M(r, \sigma)}{d r}\right)=\frac{2 G_{N}}{r^{2}} 4 \pi r^{2} \frac{M_{o} e^{-r^{2} / 4 \sigma^{2}}}{\left(4 \pi \sigma^{2}\right)^{3 / 2}}=8 \pi G_{N} \rho(r)=-8 \pi G_{N} T_{00}
$$


as expected. Notice that the sign change in (A.18) compared to eq-(2.1) is due to the choice of signature $(-,+,+,+)$ in this appendix. Similarly, one can verify that

$$
\mathcal{R}_{i j}-\frac{1}{2} g_{i j} \mathcal{R}=-8 \pi G_{N} T_{i j}
$$

by solving the covariant conservation equation of the stress energy tensor [22]

$$
\nabla_{\nu} T^{\mu \nu}=0 \Rightarrow \partial_{r} T_{r}^{r}=-\frac{1}{2} g^{00}\left(\partial_{r} g_{00}\right)\left(T_{r}^{r}-T_{0}^{0}\right)-g^{\theta \theta}\left(\partial_{r} g_{\theta \theta}\right)\left(T_{r}^{r}-T_{\theta}^{\theta}\right) .
$$

for

$$
T_{\nu}^{\mu}=\operatorname{diagonal}\left(-\rho, p_{r}, p_{\theta}, p_{\phi}\right), \quad p_{r}=-\rho, p_{\theta}=p_{\phi}=-\rho-\frac{r}{2} \partial_{r} \rho=-\rho\left(1-\frac{r^{2}}{4 \sigma^{2}}\right) .
$$

\section{Acknowledgments}

We are indebted to M. Bowers for assistance and Michael Ibison for emphasizing the importance of using absolute values. We thank Abhas Mitra, Kourosh Nozari, Eduardo Guendelman, Stephen Crothers, Jorge Mahecha, Paul Zielinski, Jack Sarfatti, Matej Pavsic for many discussions, and, especially, to Antonio Nieto, J.F Gonzalez for many insightful discussions and collaborations. Special thanks to the referee for providing very insightful remarks and references.

\section{References}

[1] A. Einstein, Sitzungsber Preuss Akad Berlin II, 831 (1915).

[2] K. Schwarzschild, Sitzungsber Preuss Akad Berlin I,189 (1916); English translation by S. Antoci and A. Loinger can be found in physics/9905030.

[3] M. Brillouin, Jour. Phys. Rad 23, 43 ( 1923); English translation by S. Antoci can be found at physics/0002009.

[4] D. Hilbert, Nachr. Ges. Wiss Gottingen Math. Phys K1, 53 (1917); H. Weyl, Ann. Physik (Leipzig) 54, 117 (1917); J. Droste, Proc. Ned. Akad. West Ser. A 19, 197 (1917).

[5] L. Abrams, Can. J. of Physics 67, 919 (1989); Physical Review D 20, 2474 (1979); Physical Review D 21, 2438 (1980); Physical Review D 21, 2941 (1980).

[6] A. Loinger, "On Black Holes and Gravitational Waves " (La Goliardica Pavese, 2002); A. Loinger and T. Marsico, "On the gravitational collapse of a massive star " physics/0512232; S. Antoci, D.E. Liebscher, " Reinstating Schwarzschild's original Manifold and its Singularity" gr-qc/0406090. 
[7] S. Crothers, Progress in Physics vol 1, 68 (2005); Progress in Physics vol 2, 3 (2005) 3; Progress in Physics vol 3, 7 (2005).

[8] N. Stavroulakis, Progress in Physics, Vol. 2, 68 (2006).

[9] M. Pavsic, Obzornik za Matematiko in Fiziko, Vol. 28, 5 (1981).

[10] S. Antoci and D.E. Liebscher, " Reinstating Schwarzschild's original Manifold and its Singularity" gr-qc/0406090.

[11] Michael Ibison, Private Communication.

[12] C. Fronsdal, Phys. Rev 116, 778 (1959); M. Kruskal, Phys. Rev 119, 1743 (1960); G. Szekers, Publ. Mat. Debreca 7, 285 (1960).

[13] P. Fiziev, "Gravitational Field of Massive Point Particle in General Relativity", gr-qc/0306088; P. Fiziev and S.V. Dimitrov, "Point Electric Charge in General Relativity" hep-th/0406077; P. Fiziev, "The Gravitational Field of Massive NonCharged Point Source in General Relativity", gr-qc/0412131; P. Fiziev, "On the Solutions of Einstein Equations with Massive Point Source", gr-qc/0407088.

[14] J.F. Colombeau, New Generalized Functions and Multiplcation of Distributions (North Holland, Amsterdam, 1984); Elementary introduction to Generalized Functions ( North Holland, Amsterdam, 1985); J. Heinzke and R. Steinbauer, " Remarks on the distributional Schwarzschild Geometry" gr-qc/0112047; R. Steinbauer and J. Vickers, "The use of generalized functions and distributions in General Relativity" gr-qc/0603078; M. Grosser, M. Kunzinger, M. Oberguggenberger, and R. Steinbauer, Geometric Theory of Generalized Functions with Applications to Relativity (Kluwer series on Mathematics and its Applications vol. 537, Kluwer, Dordrecht, 2001).

[15] H. Balasin and H. Nachbagauer, "On the distributional nature of the Energy Momentum Tensor of a Black hole or what curves the Schwarzschild Geometry" grqc/9305009; "Distributional Energy-Momentum Tensor of the Kerr-Newman SpaceTime Family" gr-qc/9312028.

[16] R. Geroch and J. Traschen, Physical Review D 36, 1017 (1987).

[17] T. Damour and H. Nicolai, "Symmetries, Singularities and the De-emergence of Spacetime" hep-th/0705.2643; T. Damour, "Cosmological Singularities and a Conjectured Gravity/Coset Correspondence" hep-th/0704.0732; T. Damour, M. Henneaux, B. Julia, and H. Nicolai, Phys. Letts B 509, 323 (2001).

[18] C. Rovelli, Quantum Gravity (Cambridge University Press, 2004); Class and Quantum Gravity, 8, 297 (1991); Class and Quantum Gravity 8, 317 (1991).

[19] T. Nakamura, " Factor two discrepancy of Hawking radiation temperature" hepth/0706.2916. 
[20] T. Ohta and R. Mann, Class.Quant.Grav. 13, 2585 (1996); R. Mann, D. Robbins, and T. Ohta, Phys. Rev. Lett. 82, 3738 (1999); F. Burnell, R. Mann, and T. Ohta, Phys. Rev. Lett. 90,134101 (2003); R. Kerner and R. Mann, Class. Quant .Grav. 20, L133 (2003).

[21] L. Lewis, Coulomb Potential of a Point in Theta Noncommutative Geometry" hepth/0605140.

[22] P. Nicolini, A. Smalagic, and E. Spallucci, Phys. Letts B 632, 547 (2006); P. Nicolini, J. Phys. A 38, L631 (2005).

[23] S. Ansoldi, P. Nicolini, A. Smalagic, and E. Spallucci, "Noncommutative Geometry Inspired Charged Black holes" gr-qc/0612035; K. Nozari and S. Mehdipour," Noncommutative Geometry inspired Charged Black Holes in Extra Dimensions", hep-th/0707.1080.

[24] T. Rizzo, JHEP 0609, 021 (2006).

[25] Q.Q. Jiang, S.Q Wu, and X. Cai, "Hawking radiation as tunneling from the Kerr and Kerr-Newman balck holes" hep-th/0512351.

[26] J. Makela and A. Peltola," Gravitation and Spacetime: The Einstein equation of State Revisited" gr-qc/0612078.

[27] S. Vacaru, P. Stavrinos, E. Gaburov, and D. Gonta, "Clifford and RiemannFinsler Structures in Geometric Mechanics and Gravity", , Selected Works Differential Geometry-Dynamical Systems Monograph (Geometry Balkan Press, 2006); S. Vacaru, Phys. Letts B 498, 74 (2001); Jour. Math Phys 46, 042503 (2005); Jour. Math Phys 46, 032901 (2005); Jour. Math Phys 47, 093504 (2006).

[28] S, Vacaru, "Nonholonomic Ricci Flows: I. Riemann Metrics and Lagrange-Finsler Geometry", math.DG/0612162.

[29] A. Kholodenko, "Towards physically motivated proofs of the Poincare' and the geometrization conjectures" hep-th/0701084.

[30] G. Perelman, "Finite extinction time for the solutions to the Ricci flow on certain three-manifolds" math.DG/0307245; "Ricci flow with surgery on three-manifolds" math.DG/0303109; "The entropy formula for the Ricci flow and its geometric applications" math.DG/0211159.

[31] I. Bakas, D. Orlando, and P. Petropoulos, "Ricci flows and expansion in axiondilaton cosmology" hep-th/0610281.

[32] T. Padmanabhan, "Dark Energy : Mystery of the Millennium" astro-ph/0603114.

[33] C. Castro, Phys. Letts B 626, 209 (2005) 209; Foundations of Physics 35, 971 (2005); Progress in Physics vol 2, 86 (2006). 
[34] C. Castro, Mod. Phys. Lett A 21, 2685 (2006); Foundations of Physics 37, 366 (2007); Mod. Phys. Lett A17, 2095 (2002).

[35] C. Castro, "Novel Remarks on Horizonless Static Spherically Symmetric Solutions of Einstein equations" CTSPS preprint, April 2006.

[36] C. Castro and A. Granik, Foundations of Physics 33, 445 (2003); C. Castro, Journal Entropy 3, 12 (2001).

[37] A. Mitra, Found. Phys. Letts 13, 543 (2000); Found. Phys. Letts 15, 439 (2002); Mon. Not. R. Astron. Soc, 369, 492 (2006).

[38] R. Wald, General Relativity (University of Chicago Press, 1984).

[39] R. Wald, Phys. Rev D 48, R3427 (1993); T. Jacobson, G. Kang, and R. Myers, "Black Hole entropy in higher curvature gravity" gr-qc/9502009.

[40] G. Horowitz, J. Maldacena, and A. Strominger, Phys. Letts B 383, 151 (1996); G. Horowitz, D. Lowe, and J. Maldacena, Phys. Rev. Lett 77, 430 (1996).

[41] K. Nozari and S. H. Mehdipour, " Failure of standard Thermodynamics in Planck Scale Back Hole System " hep-th/0610076.

[42] G. Shipov, "Dark Energy in the theory of Physical Vacuum" [http://www.shipov.com].

[43] C. Castro, J. A. Nieto, and J. F Gonzalez, "Running Newtonian coupling and horizonless solutions in Quantum Einstein Gravity" in Quantization in Astrophysics, Brownian motion and Supersymmetry ( MathTiger publishers, Chennai, India; F. Smarandache and V. Christianato, eds, 2006).

[44] A. Bonanno and M. Reuter, "Renormalization group improved black hole spacetime" hep-th/0002196; M. Reuter and J.M. Schwindt, "A Minimal Length from Cutoff Modes in Asymptotically Safe Quantum Gravity" hep-th/0511021; M. Reuter and J.M. Schwindt, " Scale-dependent structures and causal structures in Quantum Einstein Gravity " hep-th/0611294; A.Bonanno, M.Reuter " Spacetime Structure of an Evaporating Black Hole in Quantum Gravity" hep-th/0602159; Phs. Rev. D 73, 0830005 (2006).

[45] C. Castro and J. A. Nieto," On $2+2$ dimensions, Strings, Black Holes and Maximal acceleration in Phase Spaces", Int. J Mod. Phys A 22, 2021 (2007).

[46] I. Bars, Lecture at Strings 91 (Stonybrook, June 1991).

[47] E. Witten, "On black holes in string theory" Lecture given at Strings '91 (Stony Brook, June 1991) hep-th/9111052.

[48] E. Witten, Phys. Rev. D 44, 314 (1991). 
[49] J. A. Nieto, Phys. Lett A 262, 274 (1999).

[50] L. Modesto, "Evaporating loop quantum black hole" gr-qc/0612084; "Black hole interior from loop quantum gravity" gr-qc/0611043; "Gravitational collapse in loop quantum gravity" gr-qc/0610074.

[51] S. Ketov, Quantum Non-linear Sigma Models, Conformal Field Theory, Supersymmetry, Black Holes and Strings (Springer Verlag Berlin-Heidelberg, 2000).

[52] M. Green, J. Schwarz, and E. Witten, Superstring Theory ( Cambridge University Press, 1986).

[53] P. S. Aspinwall, "The Breakdown of Topology at Small Scales" JHEP 0407, 021 (2004); "A Point's Point of View of Stringy Geometry" JHEP 0301, 002 (2003).

[54] M. Lapidus and M. Frankenhuysen, Complex Dimensions and the Zeros of the Zeta Functions, (Birkhauser, New York, 2000).

[55] C. Castro, Physica A 347, 184 (2005).

[56] J. Wess, "Einstein-Riemann Gravity on Deformed Spaces" hep-th/0611025.

[57] M. Born, Proc. Royal Society A 165, 291 (1938); E. Caianiello, Lett. Nuov. Cimento 32, 65 (1981); G. Lambiase, G. Papini, and G. Scarpetta, Phys. Letts A 263, 147 (1999); M. Toller, Int. Jour. Theor. Phys 29, 963 (1990); V. Nesterenko, Class. Quan. Grav 9, 1101 (1992).

[58] H. Brandt, Contemporary Mathematics 196, 273 (1996); Chaos, Solitons and Fractals 10, 267 (1999).

[59] C. Castro, Foundations of Physics, 35, 971 (2005); Progress in Physics, 1, 20 (2006).

[60] D. Amati, M. Ciafaloni, and G. Veneziano, Phys. Letts B 197, 81 (1987); D. Gross and P. Mende, Phys. Letts B 197, 129 (1987).

[61] C. Castro, Jour. Phys A : Math. Gen 39, 14205 (2006).

[62] C.Castro, "The Charge-Mass-Spin relation of a Clifford Polyparticle and KerrNewman Black Holes Foundations of Physics 34, 107 (2004). 